\title{
THE ARECIBO LEGACY FAST ALFA SURVEY. III. H I SOURCE CATALOG OF THE NORTHERN VIRGO CLUSTER REGION
}

\author{
Riccardo Giovanelli, ${ }^{1,2}$ Martha P. Haynes, ${ }^{1,2}$ Brian R. Kent, ${ }^{1}$ Amélie Saintonge, ${ }^{1}$ Sabrina Stierwalt, ${ }^{1}$ \\ Adeel Altaf, ${ }^{3}$ Thomas Balonek, ${ }^{2,4}$ Noah Brosch, ${ }^{5}$ Shea Brown, ${ }^{6}$ Barbara Catinella, ${ }^{7}$ Amy Furniss, ${ }^{8}$ \\ Josh Goldstein, ${ }^{3}$ G. Lyle Hoffman, ${ }^{3}$ Rebecca A. Koopmann, ${ }^{2,9}$ David A. Kornreich, ${ }^{8}$ Bilal Mahmood, ${ }^{9}$ \\ Ann M. Martin, ${ }^{1}$ Karen L. Masters, ${ }^{10}$ Arik Mitschang, ${ }^{8}$ Emmanuel Momjian, ${ }^{7}$ \\ Prasanth H. Nair, ${ }^{11}$ Jessica L. Rosenberg, ${ }^{10,12}$ and Brian Walsh ${ }^{4}$ \\ Received 2006 December 7; accepted 2007 February 20
}

\begin{abstract}
We present the first installment of $\mathrm{H}$ i sources extracted from the Arecibo Legacy Fast ALFA (ALFALFA) extragalactic survey, initiated in 2005. Sources have been extracted from three-dimensional spectral data cubes exploiting a matched filtering technique and then examined interactively to yield global $\mathrm{H}$ I parameters. A total of $730 \mathrm{H}$ I detections are cataloged within the solid angle $11^{\mathrm{h}} 44^{\mathrm{m}}<$ R.A. (J2000.0) $<14^{\mathrm{h}} 00^{\mathrm{m}}$ and $+12^{\circ}<$ decl. (J2000.0) $<+16^{\circ}$ and redshift range $-1600 \mathrm{~km} \mathrm{~s}^{-1}<c z<18,000 \mathrm{~km} \mathrm{~s}^{-1}$. In comparison, the $\mathrm{H}$ I Parkes All-Sky Survey detected $40 \mathrm{H}$ I signals in the same region. Optical counterparts are assigned via examination of digital optical imaging databases. ALFALFA $\mathrm{H}$ I detections are reported for three distinct classes of signals: (1) detections, typically with $\mathrm{S} / \mathrm{N}>6.5$, (2) high-velocity clouds in the Milky Way or its periphery, and (3) signals of lower $\mathrm{S} / \mathrm{N}$ (to $\sim 4.5$ ) which coincide spatially with an optical object of known similar redshift. Although this region of the sky has been heavily surveyed by previous targeted observations based on optical flux - or size-limited samples, $69 \%$ of the extracted sources are newly reported $\mathrm{H}$ I detections. The resulting positional accuracy of $\mathrm{H}$ I sources is dependent on $\mathrm{S} / \mathrm{N}$ : it averages $24^{\prime \prime}$ $\left(20^{\prime \prime}\right.$ median) for all sources with $\mathrm{S} / \mathrm{N}>6.5$ and is of order $\sim 17^{\prime \prime}\left(14^{\prime \prime}\right.$ median) for signals with $\mathrm{S} / \mathrm{N}>12$. The median redshift of the sample is $\sim 7000 \mathrm{~km} \mathrm{~s}^{-1}$, and its distribution reflects the known local large-scale structure including the Virgo Cluster and the void behind it, the A1367-Coma supercluster at $c z \sim 7000 \mathrm{~km} \mathrm{~s}^{-1}$, and a third, more distant overdensity at $c z \sim 13,000 \mathrm{~km} \mathrm{~s}^{-1}$. Distance uncertainties in and around the Virgo Cluster perturb the derived H I mass distribution. Specifically, an apparent deficiency of the lowest $\mathrm{H}$ I mass objects can be attributed, at least in part, to the incorrect assignment of some foreground objects to the cluster distance. Several extended $\mathrm{H}$ I features are found in the vicinity of the Virgo Cluster. A small percentage (6\%) of $\mathrm{H}$ I detections have no identifiable optical counterpart, more than half of which are high-velocity clouds in the Milky Way vicinity; the remaining 17 objects do not appear connected to or associated with any known galaxy. Based on these initial results, ALFALFA is expected to fulfill, and even exceed, its predicted performance objectives in terms of the number and quality of $\mathrm{H}$ I detections.
\end{abstract}

Key words: galaxies: distances and redshifts — galaxies: halos — galaxies: luminosity function, mass function galaxies: photometry — galaxies: spiral — radio lines: galaxies

Online material: machine-readable table

\section{INTRODUCTION}

Because the evolution of the $\mathrm{H}$ i content with time promises to be a powerful tracer of galaxy evolution even into the so-called Dark Ages, it remains imperative to understand fully the true census of $\mathrm{H}$ I-bearing objects at $z \sim 0$. As spectroscopic tracers, $\mathrm{H}$ I emission-line profiles yield not only the redshift but also measures of the total $\mathrm{H}$ I mass and the radial component of the rotational velocity via the $\mathrm{H}$ I line width, thus providing quantitative insight into the gas content and total mass. At the same time

\footnotetext{
${ }^{1}$ Center for Radiophysics and Space Research, Cornell University, Ithaca, NY 14853, USA; riccardo@astro.cornell.edu, haynes@astro.cornell.edu, bkent@ astro.cornell.edu, amartin@astro.cornell.edu, amelie@astro.cornell.edu, sabrina@ astro.cornell.edu.

2 National Astronomy and Ionosphere Center, Cornell University, Ithaca, NY 14853, USA. The National Astronomy and Ionosphere Center is operated by Cornell University under a cooperative agreement with the National Science Foundation.

${ }^{3}$ Hugel Science Center, Lafayette College, Easton, PA 18042, USA; hoffmang@ lafayette.edu,goldstj@lafayette.edu, altafa@lafayette.edu.

${ }^{4}$ Department of Physics and Astronomy, Colgate University, Hamilton, NY 13346,USA; tbalonek@mail.colgate.edu, bwalsh@bu.edu.

5 The Wise Observatory and The School of Physics and Astronomy, Raymond and Beverly Sackler Faculty of Exact Sciences, Tel Aviv University, Israel; noah@wise.tau.ac.il.
}

that wide-area optical (e.g., Sloan Digital Sky Survey [SDSS]; York et al. 2000) and near-infrared (e.g., Two Micron All Sky Survey [2MASS]; Skrutskie et al. 2006) surveys have been cataloging millions of galaxies, the $\mathrm{H}$ I Parkes All-Sky Survey (HIPASS; Barnes et al. 2001) produced the first wide-area blind extragalactic $\mathrm{H}$ I line survey. HIPASS covered $\sim 30,000 \mathrm{deg}^{2}$, producing a final catalog (Meyer et al. 2004) of $4315 \mathrm{H} \mathrm{I}$ detections south of decl. $=+2^{\circ}$, and another 1002 (Wong et al. 2006) for $+2^{\circ}<$ decl. $<+20^{\circ}$. Because HIPASS was limited in depth and resolution, it did not sample an adequate volume to

\footnotetext{
${ }^{6}$ Astronomy Department, University of Minnesota, Minneapolis, MN 55455, USA; brown@astro.umn.edu.

${ }^{7}$ Arecibo Observatory, National Astronomy and Ionosphere Center, Arecibo, PR 00612, USA; bcatinel@naic.edu, emomjian@naic.edu.

${ }^{8}$ Department of Physics, Humboldt State University, Arcata, CA 95521, USA; dak24@humboldt.edu.

${ }^{9}$ Department of Physics and Astronomy, Union College, Schenectady, NY 12308, USA; koopmanr@union.edu, mahmoodb@union.edu.

${ }^{10}$ Harvard-Smithsonian Center for Astrophysics, Cambridge, MA 02138-1516, USA; kmasters@cfa.harvard.edu.

${ }_{11}$ Astronomy Department, Indiana University, Bloomington, IN 47405, USA; phnair@astro.indiana.edu.

${ }^{12}$ Current address: George Mason University, Fairfax, VA 22030, USA; jrosenb4@gmu.edu.
} 
yield a "cosmologically fair" sample of the universe; the median redshift of HIPASS sources is $\sim 2800 \mathrm{~km} \mathrm{~s}^{-1}$, near the outer boundary of the Local Supercluster. Of critical importance for the determination of the faint-end slope of the $\mathrm{H}$ i mass function (HIMF), only a handful of galaxies of very low $\mathrm{H}$ i mass were detected, and those were so near the Milky Way that distance uncertainties make their $\mathrm{H}$ I masses likewise highly uncertain (Masters et al. 2004).

$\mathrm{H}$ I line surveys are of special importance when combined with optical/IR surveys so that both the stellar and gaseous components are sampled. As was shown also by earlier studies such as the Arecibo Dual Beam Survey (ADBS; Rosenberg \& Schneider 2002), blind H I surveys detect a population of low surface brightness (LSB), gas-rich objects that are often missed by magnitudelimited optical and near-IR samples. Likewise, not all galaxies exhibit detectable $\mathrm{H}_{\mathrm{I}}$ line emission, and $\mathrm{H}_{\mathrm{I}}$ surveys are limited to some degree by their positional accuracy and angular resolution since the telescopes involved have relatively large beam sizes. A true accounting of the extragalactic census must incorporate full sampling of the population of gas-rich objects sampled by $\mathrm{H}$ I surveys in complement to the stellar-rich ones detected by surveys like SDSS and 2MASS.

Based on both the success of HIPASS and its limitations, we have initiated a "second-generation" $\mathrm{H}_{\mathrm{I}}$ blind survey, the Arecibo Legacy Fast ALFA (ALFALFA) survey, which exploits the availability of the new multibeam Arecibo L-band Feed Array (ALFA) on the $305 \mathrm{~m}$ antenna. ALFALFA will require $\sim 4000 \mathrm{hr}$ of telescope time to survey $\sim 7000 \mathrm{deg}^{2}$ of the high Galactic latitude sky visible from Arecibo (Giovanelli et al. 2005a, hereafter Paper I). HIPASS used a 13 beam receiver on the Parkes $62 \mathrm{~m}$ telescope (15.5' half-power beamwidth); ALFALFA exploits a similar sevenfeed receiver array on the $305 \mathrm{~m}$ Arecibo telescope $\left(\sim 3.5^{\prime}\right)$. The survey design of ALFALFA exploits the superior collecting area and angular resolution of the Arecibo telescope, as well as the broader bandwidth and spectral resolution of its digital correlator. Furthermore, ALFALFA makes use of automated signal-detection techniques to produce source catalogs unbiased by the width of the H I signal (Saintonge 2007). Designed to surpass significantly the H I survey benchmark established by HIPASS, ALFALFA improves on its predecessor by a factor of $\sim 8$ in sensitivity, $\sim 4$ in angular resolution, $\sim 3$ in spectral resolution, and $\sim 1.6$ in spectral coverage. The added depth of ALFALFA will allow it to sample a "fair" volume of the universe. In particular, ALFALFA will sample well the volume corresponding to the so-called convergence depth (Giovanelli et al. 1998; Dale \& Giovanelli 2000), which contributes most of the peculiar velocity of the Milky Way with respect to the cosmic microwave background.

Simulations presented in Paper I predicted that ALFALFA would detect some 20,000 extragalactic $\mathrm{H}_{\mathrm{I}}$ line sources, from very nearby low-mass dwarfs to massive spirals at $z \sim 0.06$. The survey is designed specifically to determine robustly the HIMF in the local universe and will at the same time provide a census of $\mathrm{H}$ I in the surveyed sky area, making it especially useful in synergy with other wide-area surveys such as SDSS, 2MASS, Galaxy Evolution Explorer, and Akari. The full scope and goals of the survey are described further in Paper I.

$\mathrm{H}$ I line surveys yield three principal observational parameters: the integrated $\mathrm{H}_{\mathrm{I}}$ line flux, the systemic redshift, and the Doppler line width. For resolved objects, they can also yield an estimate of the $\mathrm{H}$ I size and, if the source fills the beam, the H I column density. ALFALFA is expected to resolve about 500 nearby galaxies; other objects with exceptionally extended $\mathrm{H}$ I distributions will also be mapped. Its positional accuracy (see $\S 5$ ) allows identification of the optical counterparts of the $\mathrm{H}$ I detections by immediate cross- reference with the large optical/IR imaging databases. The most interesting objects will perhaps be the isolated $\mathrm{H}$ i sources which may not have optical counterparts, the so-called dark galaxies.

The ALFALFA survey was initiated in 2005 February. Since then, we have conducted observations regularly and anticipate allocations of 700-900 hr of telescope time per year. In order to provide timely information to the community, a public Web site ${ }^{13}$ is updated regularly to provide survey plans and status information. Because of the two-pass drift mode observing strategy (Paper I), complete spectral coverage combines many separate data sets obtained over observing periods spread over many months. Final three-dimensional cubes from which signals can be reliably extracted cannot be produced until the entire data set covering a targeted region is acquired. In fact, the first-year data sets were rather incomplete, so construction of full-coverage threedimensional data cubes has been possible only recently.

Given the area of sky visible to the Arecibo telescope, a prime target for ALFALFA is the Virgo Cluster and its surroundings. Although the spirals in the core of the Virgo Cluster are strongly H I-deficient (Davies \& Lewis 1973; Chamaraux et al. 1980; Giovanelli \& Haynes 1983; Solanes et al. 2002), the cluster periphery contains many known optical late-type galaxies. ALFALFA is specifically designed to detect objects of relatively low $\mathrm{H}$ I mass, $\sim 3 \times 10^{7} M_{\odot}$, at the Virgo distance. Hence, the Virgo region of the sky was targeted by ALFALFA from the beginning; its central region has been the first zone to be surveyed completely. In this paper, we present a catalog of $\mathrm{H}$ i sources extracted from two constant-declination strips of three-dimensional grids, covering a swath $4^{\circ}$ wide in declination $+12^{\circ}<\operatorname{decl}$. (J2000.0) $<$ $+16^{\circ}$ and stretching $33^{\circ}$ in right ascension (R.A.), $11^{\mathrm{h}} 44^{\mathrm{m}}<$ R.A. (J2000.0) $<14^{\mathrm{h}} 00^{\mathrm{m}}$. This area includes the northern section of the Virgo Cluster. With a complete data set now in hand for a first installment covering $132 \mathrm{deg}^{2}$, this paper presents the first ALFALFA $\mathrm{H}$ i detection catalog, in the spirit of prompt access that the legacy nature of the survey promises. A catalog containing sources of the southern part of the Virgo Cluster region is in preparation (B. R. Kent et al. 2007, in preparation).

In $\S 2$ we discuss the ALFALFA survey observations and data analysis. In $\S 3$ we briefly discuss issues related to the data quality of the region sampled by the catalog, as well as signal extraction criteria. The contents of the catalog are also described. Section 4 gives an overview of the statistical properties of the cataloged sample. The positional accuracy of the $\mathrm{H}$ I positions is discussed in $\S 5$. Section 6 summarizes the $\mathrm{H}$ I detections presented here and what the results of this first installment predict for the future results of the full ALFALFA survey. A Hubble constant of $70 \mathrm{~km} \mathrm{~s}^{-1} \mathrm{Mpc}^{-1}$ is used for distance-dependent calculations unless otherwise specified.

\section{OBSERVATIONS AND DATA REDUCTION}

ALFALFA uses the seven-feed ALFA receiver system and a spectral-line back end capable of instantaneously producing spectra from the two linear polarizations of each beam and covering a bandwidth of $100 \mathrm{MHz}$. The angular resolution of the survey is given by the elliptical shape of each of ALFA's beams, $3.3^{\prime} \times 3.8^{\prime}$ (Paper I), and the spectral resolution is $25 \mathrm{kHz}$, which translates to about $5.5 \mathrm{~km} \mathrm{~s}^{-1}$ at $c z \sim 0 \mathrm{~km} \mathrm{~s}^{-1}$ before spectral smoothing is applied. ALFALFA surveys the sky with the telescope in "almost fixed azimuth" drift mode: the telescope azimuth arm is placed on the meridian, and the sky drifts by. Small adjustments of the zenith angle are applied throughout the observing period to maintain the beam tracks at constant J2000.0 declination.

\footnotetext{
${ }^{13}$ Available at http://egg.astro.cornell.edu/alfalfa.
} 
Each region of sky is visited twice, at two epochs spaced by a few months in Earth's orbit about the Sun. No Doppler tracking of the local oscillator frequency is employed, so that cosmic signals shift in frequency between the two epochs by the difference in their heliocentric velocities, projected along the line of sight. The resulting coverage yields parallel tracks of constant J2000.0 declination, separated from one another by $1.05^{\prime}$. The sampling rate in R.A. is $1 \mathrm{~Hz}$. In both coordinates, the sampling is significantly better than the Nyquist rate. Data-taking for ALFALFA was initiated in 2005 February, and in the practical context of time allocation at a widely used, multidisciplinary national facility like Arecibo, completion of the full survey is projected to require about 6 yr. More technical detail regarding the equipment, observing mode, and sensitivity issues can be found in Paper I.

The ALFALFA data-processing scheme has been briefly outlined in Paper I and will be described in detail elsewhere (R. Giovanelli et al. 2007, in preparation; Saintonge 2007). Full processing of all survey data to level I, which includes bandpass calibration, radio frequency interference (RFI) flagging, continuum-source identification, and extraction of drift scans, is carried out shortly after data taking, as anticipated in Paper I. However, the production of three-dimensional data cubes that fully sample a region of sky requires the completion of both passes and thus cannot be completed until all data covering that region are in hand. In Paper I we discussed the parceling of the sky into data-processing units we referred to as tiles, of $10^{\mathrm{m}}$ in R.A. by $4^{\circ}$ in declination. In practice, as the processing needs demanded a distributed strategy, data units of smaller size than the above mentioned tiles were found to be compatible with a minimum denominator computer performance. The data units thus adopted each cover a sky area of $2.4^{\circ} \times 2.4^{\circ}$ in R.A. and declination; in order to avoid confusion with the previous definition of tiles, we refer to regularly gridded data cubes of $2.4^{\circ} \times 2.4^{\circ}$ and preset centers as grids. Centers of grids that are adjacent in R.A. are separated from each other by $8^{\mathrm{m}}$; the separation in declination is $2^{\circ}$. A spatial overlap of about $20 \%$ in each coordinate allows effective spatial coverage of all sources found in the overlap regions. In order to maintain the gridded data file size at a limit comfortably manipulated by modest computer hardware, we break each spatial grid into four overlapping spectral subgrids of 1024 channels each, after discarding the band-ends. Because of RFI, the spectral resolution is not reduced at this stage, and the full information on spectral weights, as described in $\S 2.3$ below, is retained. Each 1024 channel three-dimensional spectral cube has a final size of 380 Mbytes.

\subsection{Access to Data Products}

In the interest of timely access to our data products by the community, we plan to release source catalogs at the earliest possible time, consistent with the legacy character of ALFALFA. The catalog presented here is the first part of an archival database being developed as a collaborative project with the National Astronomy and Ionosphere Center and the Cornell Theory Center. The data catalog products are available online as a node of the US National Virtual Observatory. ${ }^{14}$ Included already at that Web site are catalogs and spectral data products of targeted single-beam $\mathrm{H}_{\mathrm{I}}$ observations of $\sim 9000$ galaxies observed by our group (Springob et al. 2005) and the ALFALFA precursor observations (Giovanelli et al. 2005b, hereafter Paper II). At the time of publication of this paper, the catalog presented here and access to the spectral profiles

\footnotetext{
14 Available at http://arecibo.tc.cornell.edu/hiarchive. This research has made use of data obtained from or software provided by the US National Virtual Observatory, which is sponsored by the National Science Foundation.
}

associated with the $\mathrm{H}$ I detections will be incorporated into our existing $\mathrm{H}$ i digital archive. ${ }^{15}$ An ongoing development effort aims its focus on the protocol for long-term public delivery of the threedimensional ALFALFA data set through Web-based access tools. At this time, delivery of the three-dimensional data is made possible through the observing team itself, by direct contact to R. G. or M. P. H. A major challenge is data volume; each of the threedimensional cubes covering the 34 individual grids constituting the current catalog requires 1.5 Gbytes. Allowing access and manipulation of the gridded data publicly will require the development of distributed computational tools.

In this work, we present a catalog of $\mathrm{H}_{\text {I }}$ sources extracted from the ALFALFA grids covering a region stretching from $+12^{\circ}$ to $+16^{\circ}$ in declination and from $11^{\mathrm{h}} 44^{\mathrm{m}}$ to $14^{\mathrm{h}} 00^{\mathrm{m}}$ in R.A. For reference to our database, the denominations of the grids constituting this catalog are $1148+13$ to $1356+13$ and $1148+15$ to $1356+15$, in steps of $8^{\mathrm{m}}$ in R.A. The solid angle subtended by this region is $\sim 132 \mathrm{deg}^{2}$, which is $\sim 1.9 \%$ of the sky to be ultimately surveyed by ALFALFA. The coverage of the region is complete by the target goals of ALFALFA, i.e., the region has been sampled by two separate passes with the ALFA array in drift mode. The average quality of the data is fair to excellent; details on the data quality at a particular location can be garnered by consultation with any of the Cornell authors or by consultation of the ALFALFA Web site mentioned in footnote 13. This type of information will eventually be accessible through our NVO-compliant node.

\subsection{Impact of $M 87$}

The surveyed region includes the northern part of the Virgo Cluster and M87, at R.A. $=+12^{\mathrm{h}} 30^{\mathrm{m}} 49.4^{\mathrm{s}}$, decl. $=12^{\circ} 23^{\prime} 28^{\prime \prime}$ (J2000.0). The very strong radio source associated with M87 has a $1.4 \mathrm{GHz}$ continuum flux of $220 \mathrm{Jy}$, which increases the system temperature of the Arecibo telescope at $\mathrm{L}$ band by a factor of 70 when M87 crosses within the main beam of the telescope. Even when M87 is detected only through the near sidelobe structure of the beam, the sensitivity decreases drastically both because of the increased system temperature and because of the onset of strong spectral standing waves. Effectively, a region of about $1^{\circ}-1.5^{\circ}$ radius centered on M87 remains inaccessible to $\mathrm{H}$ i spectroscopy except for the very brightest $\mathrm{H}$ I sources, as we illustrate in $\S 4$.

\subsection{Radio Frequency Interference}

In practice, RFI contaminates certain regions of the observed spectral window. The data-processing pipeline tracks the flagging from visual inspection of spectral samples that are deemed to be contaminated and assigns a weight to each pixel in threedimensional space according to the degree of this flagging. The quality of the spectral coverage is exemplified in Figure 1, which shows the average "spectral weight" over the full region sampled by the present source catalog. A spectral weight of 1.0 indicates good data quality and full utilization of all the data at that frequency or cosmological recession velocity. We compute an array of spectral weights, which is a quality estimator, for each spatial pixel of the survey. The drops in the spectral weights at both ends of the graph in Figure 1 arise from the diminished sensitivity found at the bandpass edges resulting from instrumental effects. The deep trough between 15,000 and $16,000 \mathrm{~km} \mathrm{~s}^{-1}$ is due to RFI originating from the Federal Aviation Administration (FAA) radar operating at $1350 \mathrm{MHz}$ near the Luis Muñoz Marin airport in San Juan. The narrower features at 8800, 3300, and

\footnotetext{
${ }^{15}$ Available at http://arecibo.tc.cornell.edu/hiarchive/alfalfa.
} 


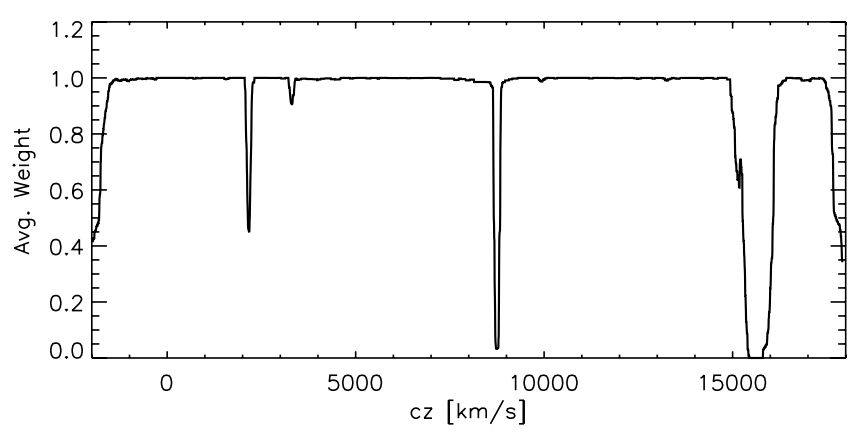

FIG. 1.-Average spectral weights for the region of this catalog, plotted vs. heliocentric recessional velocity. The steep drop-off at each end of the spectral domain is an instrumental effect associated with the spectrometer bandpass edge. The other strong troughs are associated with RFI originating with the San Juan FAA radar operating at $1350 \mathrm{MHz}$.

$2200 \mathrm{~km} \mathrm{~s}^{-1}$ are modulations of the $1350 \mathrm{MHz}$ radar signal occurring within the spectrometer. While the feature between 15,000 and $16,000 \mathrm{~km} \mathrm{~s}^{-1}$ renders inaccessible a larger cosmic volume, the feature at $8800 \mathrm{~km} \mathrm{~s}^{-1}$ affects a larger number of possible detections, given its spectral location in vicinity of the peak of the redshift distribution for our catalog. On average, about $85 \%$ of the spectral bandpass is clear for astronomical use.

The presence of RFI causes distinct volumes of the universe, which correspond to the frequency ranges of redshifted $\mathrm{H} \mathrm{I}$, to be obscured to ALFALFA. In order to maintain a proper census of the survey volume, we use the three-dimensional "pixel mask," which records the spectral weights described above.

\section{SOURCE CATALOG}

\subsection{Tabulated Data}

Shortly after each observing session, the individual $600 \mathrm{~s}$ drift scans are processed to Level I; that is, they are bandpass subtracted and calibrated, and flagging of channels affected by RFI is performed (see Paper I). Once a region of the sky has been fully sampled by ALFALFA, a three-dimensional grid is then generated from all of the individual drift scans that cross it. In practice, the band edges are dropped, and four spectral subgrids, each comprising 1024 channels, with $\sim 100$ channel overlap of each, are constructed for each $2.4^{\circ} \times 2.4^{\circ}$ spatial grid. Each spectral subgrid is then flat-fielded (i.e., baselined) and made ready for source extraction. An automatic signal extractor described in Saintonge (2007) that operates in the Fourier domain produces a preliminary catalog of sources to a specified threshold in signal-to-noise ratio $(\mathrm{S} / \mathrm{N})$. Each candidate source is then examined visually and a decision on its inclusion in a final catalog, as well as a detailed, interactively obtained set of measurements yielding the principal source parameters, is made. A spatially integrated spectrum of the source and a postage-stamp three-dimensional section of the grid centered on the source are archived.

After $\mathrm{H}$ I source candidates are inspected, measured and cataloged, identification of possible optical counterparts is carried out through inspection of the SDSS, ${ }^{16}$ Digital Sky Survey 2 via

\footnotetext{
${ }^{16}$ Funding for the SDSS has been provided by the Alfred P. Sloan Foundation, the Participating Institutions, the National Aeronautics and Space Administration, the National Science Foundation, the US Department of Energy, the Japanese Monbukagakusho, and the Max Planck Society. The SDSS Web site is http://www.sdss.org. The SDSS is managed by the Astrophysical Research Consortium for the Participating Institutions. The Participating Institutions are the University of Chicago, Fermilab, the Institute for Advanced Study, the Japan Participation Group, the Johns Hopkins University, Los Alamos National Laboratory, the Max Planck Institute for Astronomy, the Max Planck Institute for Astrophysics, New Mexico State University, the University of Pittsburgh, Princeton University, the US Naval Observatory, and the University of Washington.
}

Skyview, ${ }^{17} \mathrm{NED},{ }^{18}$ and our privately maintained database of extragalactic sources (the Arecibo General Catalog $[\mathrm{AGC}]$ ). For the vast majority of $\mathrm{H}$ i source candidates of fair to excellent $\mathrm{S} / \mathrm{N}$, a relatively unambiguous identification of an optical counterpart is possible, thanks to the positional accuracy of the $\mathrm{H}$ i sources, as described in $\S 5$. In our catalog we distinguish between three kinds of sources: (1) reliable, extragalactic $\mathrm{H}$ I candidate sources, down to a $\mathrm{S} / \mathrm{N}$ threshold of approximately $\mathrm{S} / \mathrm{N}=6.5$ (the limit is soft, as described further below), (2) high $\mathrm{S} / \mathrm{N}$ features of low velocity likely to be Galactic or perigalactic high-velocity clouds (HVCs), and (3) candidate $\mathrm{H}$ I sources of lower $\mathrm{S} / \mathrm{N}$ (approximately $4.5-$ 6.5 ), corroborated by the vicinity of a possible optical counterpart of the same redshift. The signal-extraction process also yields candidate sources of lower $\mathrm{S} / \mathrm{N}$ than those listed in the present catalog. A list of those objects is available on request. As discussed in Paper I, the most efficient strategy for confirming follow-up observations requires a high sky density of sources spread in $c z$ but at similar declination. Possible detections of low $\mathrm{S} / \mathrm{N}$ will be observed in follow-up corroborating runs, which will help us quantify the reliability, i.e., the fraction of candidate sources which observations will confirm as real, as a function of the measured $\mathrm{S} / \mathrm{N}$. Those observations will also deliver a significant addition of reliable detections, at a very low cost of telescope time per source (see Papers I and II). Figure 2 shows a few spectra of different types as described above (see figure caption and col. [14] of Table 1 for details).

A few details should be kept in mind in the use of the contents of Table 1, as described below:

Several objects straddle or are confused by the $\mathrm{H}$ I emission from the Milky Way. A spectrum of the object is generally obtained by subtracting a median spectrum obtained over a square perimetric segment of pixels around the source, from the inner part of the perimeter populated by the source. In some cases, interpolation of the spectrum across the Milky Way emission provides an adequate result. Sources for which these intrusive techniques are applied are noted in the footnotes to the machine-readable version of Table 1.

Sources are often detected in the spectral vicinity of RFI features. Interpolation across the region affected by RFI is often necessary. While the detection of the source may be clear, accurate measurement of its parameters may be impossible, and much caution should be adopted in their use. This problem is frequent for sources between $c z \sim 8500$ and $8900 \mathrm{~km} \mathrm{~s}^{-1}$, a spectral region not far removed from the median redshift of our catalog. Each of these cases is noted in the footnotes to Table 1.

Extended sources containing multiple concentrations, obviously physically associated, are sometimes found. We adopt the practice of measuring separately each of the concentrations and report them in Table 1 as separate entries. They are also noted in the footnotes to Table 1 .

Table 1 contains the principal parameters of $\mathrm{H}$ I candidate detections, namely:

Col. (1).- An entry number for this catalog.

Col. (2).-The source number in the AGC, a private database of extragalactic objects maintained by M. P. H. and R. G. The AGC entry normally corresponds to the optical counterpart except

\footnotetext{
${ }^{17}$ Skyview was developed and maintained under NASA ADP grant NAS532068 under the auspices of the High Energy Astrophysics Science Archive Research Center at the Goddard Space Flight Center laboratory of NASA.

${ }^{18}$ The NASA/IPAC Extragalactic Database is operated by the Jet Propulsion Laboratory, California Institute of Technology, under contract with the National Aeronautics and Space Administration.
} 

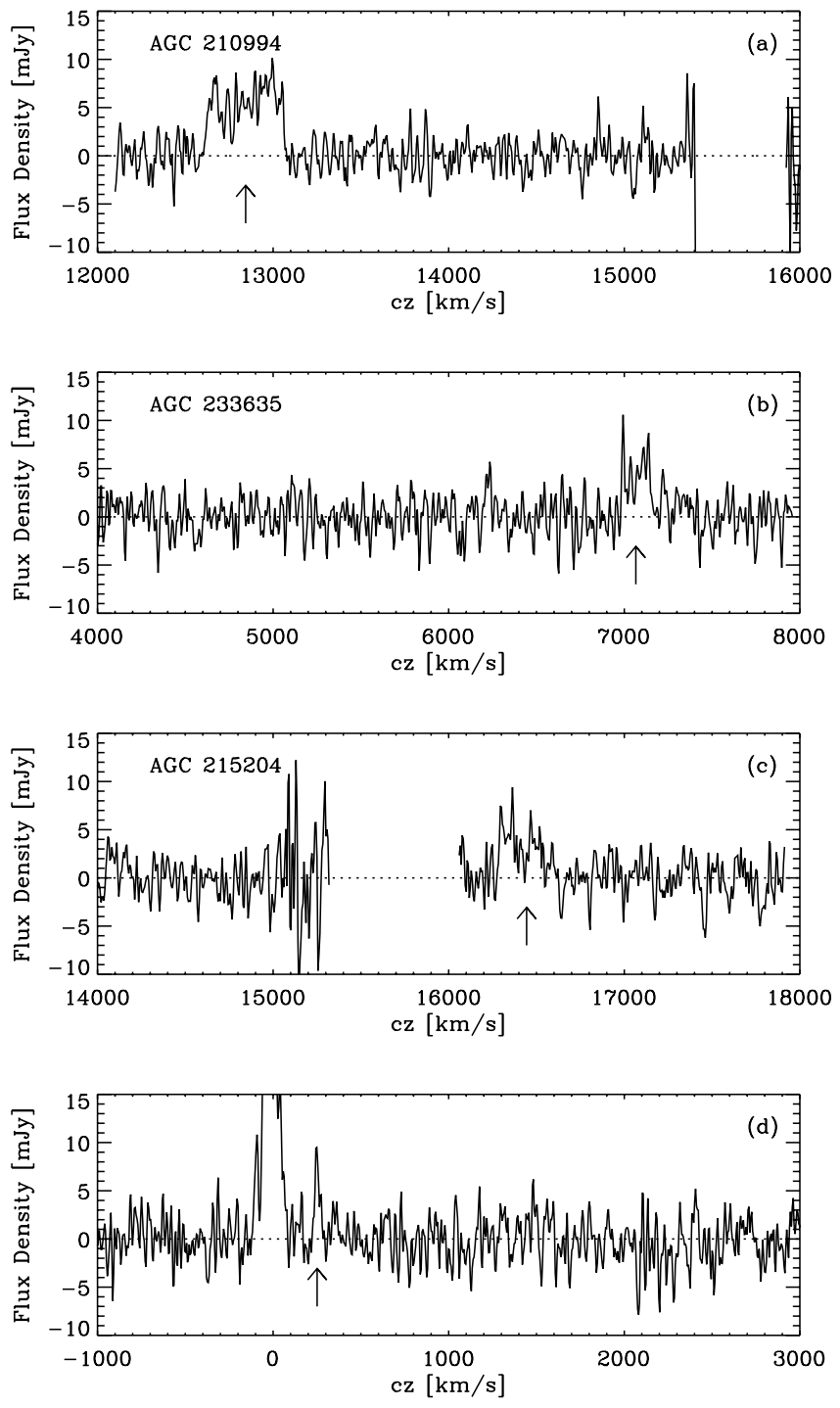

FIG. 2.-Four H I spectra showing examples of the candidate detection types described in the text (see second paragraph of $\S 3$ and description of col. [14] of Table 1 for reference): panel $a$ represents an object of $\mathrm{S} / \mathrm{N}=6.5$, a clear, well defined detection of code $=1$; panel $b$ illustrates a candidate near the lower limit of comfortable detection, with $\mathrm{S} / \mathrm{N}=6.7$, a marginal code $=1$; panel $c$ contains an example of a detection candidate of $\operatorname{code}=2$, with $\mathrm{S} / \mathrm{N}=5.5$, which is corroborated by an optical counterpart of like redshift; panel $d$ is a candidate detection with no known optical counterpart and $\mathrm{S} / \mathrm{N}=5.0$, too low to be included in this paper's catalog, but a possible target for follow-up observations. The impact of RFI can also be seen in panels $a$ and $c$.

in the cases of $\mathrm{H}$ I sources that cannot be associated with an optical object with any high degree of probability.

Cols. (3) and (4).-Center (J2000.0) of the H I source, after correction for systematic telescope pointing errors, which range between a few and about a dozen arcseconds, as described in $\S 5$. The accuracy of the $\mathrm{H}$ i positions depends on source strength. On average, the positional accuracy is about $24^{\prime \prime}$. See $\S 5$ for details.

Cols. (5) and (6).-Center (J2000.0) of the optical galaxy found to provide a reasonable optical counterpart. This position has been checked for each listed object and assessed using tools provided through the SDSS and Skyview Web sites. Quality of centroids is estimated to be $2^{\prime \prime}$ or better. The assessment of identification between optical and $\mathrm{H}$ I sources is based on spatial proximity, redshift (if optical is available), morphology, color, and evidence for optical emission lines (if known). For sources with no discernible optical counterpart and those for which such assignment is ambiguous, due to the presence of more than one equally possible optical counterpart, no optical position is listed. The latter set includes HVCs. For objects with more than one possible candidate as an optical counterpart but such that one of the candidates is significantly more preferable than the others, an optical identification is made; however, a comment on the possible ambiguity is added in the notes to this table, as indicated by an asterisk in column (14).

Col. (7).-Heliocentric velocity of the $\mathrm{H}$ i source, $c z_{\odot}$, measured as the midpoint between the channels at which the flux density drops to $50 \%$ of each of the two peaks (or of one, if only one is present) at each side of the spectral feature. Units are $\mathrm{km} \mathrm{s}^{-1}$. The error on $c z_{\odot}$ to be adopted is half the error on the width, tabulated in column (8).

Col. (8).- Velocity width of the source line profile, $W 50$, measured at the $50 \%$ level of each of the two peaks, as described for column (7). This value is corrected for instrumental broadening. No corrections due to turbulent motions, disk inclination, or cosmological effects are applied. Between brackets we show the estimated error on the velocity width, $\epsilon_{w}$, in $\mathrm{km} \mathrm{s}^{-1}$. This error is the sum in quadrature of two components: the first is a statistical error, principally dependent on the $\mathrm{S} / \mathrm{N}$ of the feature measured; the second is a systematic error associated with the subjective guess with which the observer estimates the spectral boundaries of the feature: maximum and minimum guesses of the spectral extent of the feature are flagged and the ratio of those values is used to estimate systematic errors on the width, the velocity, and the flux integral. In the majority of cases, the systematic error is significantly smaller than the statistical error; thus, the former is ignored.

Col. (9).--Integrated flux density of the source, $F_{c}$, in Jy km s ${ }^{-1}$. This is measured on the integrated spectrum, obtained by spatially integrating the source image over a solid angle of at least $7^{\prime} \times 7^{\prime}$ and dividing by the sum of the survey beam values over the same set of image pixels (see Shostak \& Allen 1980). Estimates of integrated fluxes for very extended sources with significant angular asymmetries can be misestimated by our algorithm, which is optimized for measuring sources comparable with or smaller than the survey beam. A special catalog with parameters of extended sources will be produced after completion of the survey. The estimated uncertainty of the integrated flux density, in Jy $\mathrm{km} \mathrm{s}^{-1}$ is given between brackets. Uncertainties associated with the quality of the baseline fitting are not included; an analysis of that contribution to the error will be presented elsewhere for the full survey. See description of column (8) for the contribution of a possible systematic measurement error.

Col. (10). - $\mathrm{S} / \mathrm{N}$ of the detection, estimated as

$$
\mathrm{S} / \mathrm{N}=\left(\frac{1000 F_{c}}{W 50}\right) \frac{w_{\mathrm{smo}}^{1 / 2}}{\sigma_{\mathrm{rms}}}
$$

where $F_{c}$ is the integrated flux density in Jy km s${ }^{-1}$, as listed in column (9), the ratio $1000 F_{c} / W 50$ is the mean flux across the feature in mJy, $w_{\text {smo }}$ is either $W 50 /(2 \times 10)$ for $W 50<400 \mathrm{~km} \mathrm{~s}^{-1}$ or $400 /(2 \times 10)=20$ for $W 50 \geq 400 \mathrm{~km} \mathrm{~s}^{-1}$ ( $w_{\text {smo }}$ is a smoothing width expressed as the number of spectral resolution bins of $10 \mathrm{~km} \mathrm{~s}^{-1}$ bridging half of the signal width), and $\sigma_{\mathrm{rms}}$ is the rms noise figure across the spectrum measured in mJy at $10 \mathrm{~km} \mathrm{~s}^{-1}$ resolution, as tabulated in column (11). In a similar analysis, in Paper II we adopted a maximum smoothing width $W 50 / 20=10$. See Figure 6 and related text below for details. The value of the smoothing width could be interpreted as an indication of the degree to which spectral smoothing aids in the visual detection 
of broad signals, against broadband spectral instabilities. The ALFALFA data quality appears to warrant a more optimistic adoption of the smoothing width than previously anticipated.

Col. (11).-Noise figure of the spatially integrated spectral profile, $\sigma_{\mathrm{rms}}$, in $\mathrm{mJy}$. The noise figure as tabulated is the rms and measured over the signal- and RFI-free portions of the spectrum, after Hanning smoothing to a spectral resolution of $10 \mathrm{~km} \mathrm{~s}^{-1}$.

Col. (12). - Adopted distance in Mpc, $D_{\mathrm{Mpc}}$. For objects with $c z_{\mathrm{CMB}}>3000$, the distance is simply $c z_{\mathrm{CMB}} / H_{0} ; c z_{\mathrm{CMB}}$ is the recession velocity measured in the cosmic microwave background reference frame and $H_{0}$ is the Hubble constant, for which we use a value of $70 \mathrm{~km} \mathrm{~s}^{-1} \mathrm{Mpc}^{-1}$. For objects of lower $c z_{\mathrm{CMB}}$, we use a peculiar velocity model for the local universe, as described in Paper II. Objects that are thought to be parts of clusters or groups are assigned the $c z_{\mathrm{CMB}}$ of the cluster or group. Cluster and group membership are assigned following the method described in Springob et al. 2006. A detailed analysis of group and cluster membership of ALFALFA objects will be presented elsewhere. Note that the Virgo Cluster extends over much of the solid angle surveyed. This introduces unavoidable ambiguities in the distance assignment, as the peculiar flow model only corrects for large-scale perturbations in the velocity field and is unable to deal effectively with regions in the immediate vicinity of the cluster and along a section of a cone which contains the cluster, up to $c z \sim 2500 \mathrm{~km} \mathrm{~s}^{-1}$. The distance to the Virgo Cluster was assumed to be $16.7 \mathrm{Mpc}$.

Col. (13).- - Logarithm in base 10 of the $\mathrm{H}$ I mass, in solar units. That parameter is obtained by using the expression $M_{\mathrm{H} \mathrm{I}}=2.356 \times 10^{5} D_{\mathrm{Mpc}}^{2} F_{c}$.

Col. (14).-Object code, defined as follows:

Code 1 refers to sources of $\mathrm{S} / \mathrm{N}$ and general qualities that make it a reliable detection. By general qualities we mean that, in addition to an approximate $\mathrm{S} / \mathrm{N}$ threshold of 6.5 , the signal should exhibit a good match between the two independent polarizations and a spatial extent consistent with expectations given the telescope beam characteristics. Thus, some candidate detections with $\mathrm{S} / \mathrm{N}>6.5$ have been excluded on grounds of polarization mismatch, spectral vicinity to RFI features, or peculiar spatial properties. Likewise, some features of $\mathrm{S} / \mathrm{N}<6.5$ are included as reliable detections, due to optimal overall characteristics of the feature. The $\mathrm{S} / \mathrm{N}$ threshold for acceptance of a reliable detection candidate is thus soft. In a preliminary fashion, we estimate that detection candidates with $\mathrm{S} / \mathrm{N}>6.5$ in Table 1 are reliable; i.e., they will be confirmed in follow-up observations in better than $95 \%$ of cases (Saintonge 2007). Follow-up observations planned for 2007 will set this estimate on stronger statistical grounds.

Code 2 refers to sources of low $\mathrm{S} / \mathrm{N}(<6.5)$, which would ordinarily not be considered reliable detections by the criteria set for code 1 . However, those $\mathrm{H}$ I candidate sources are matched with optical counterparts with known optical redshifts that match those measured in the $\mathrm{H}$ I line. These candidate sources, albeit detected by our signal finding algorithm, are accepted as likely counterparts only because of the existence of previously available, corroborating optical spectroscopy. We refer to these sources as "priors." We include them in our catalog because they are very likely to be real.

Code 9 refers to objects assumed to be HVCs; no estimate of their distances is made. Figure 2 shows a few examples of candidate detections.

Notes flag.- An asterisk in column (14) indicates that a comment is included for this source in the text below.

Only the first few entries of Table 1 are listed in the printed version of this paper. The full contents of Table 1 are accessible in the electronic version of the Astronomical Journal and are also available through our public digital archive site.

\subsection{Notes to Tabulated Data}

Notes associated with the objects listed in Table 1 follow. Each note is preceded by the catalog entry name as listed in column (1) of Table 1.

1-2.-Extended HVC?

1-20.-Also possible optical identification with companion at $114802.6+145228$;

1-21.-Blend with AGC 210799, parameters very uncertain;

1-22.-Blend with AGC 212846, parameters very uncertain;

1-23.-Emission of AGC 210799 also in spectrum;

1-28.-Optical identification uncertain as 114904.4+133746; also possibly $114858.8+133710$, nearer to $\mathrm{H}$ I but $\sim 1$ mag fainter;

1-36. - Possible blend; alternative optical identification is $115012.8+154620$; opt $z=0.045$, which is 1 mag brighter but twice as far from $\mathrm{H}$ i center;

1-42. - Extended $\mathrm{H}$ I, blended with emission to the northeast;

1-44.-No identifiable optical counterpart; appendage of AGC $215231 ?$

1-46.-H I emission merges in spectral region with strong RFI; H I parameters very uncertain;

1-62.-No clear optical counterpart; UGC 6911 5.3' to west, similar $c z$; H I feature appears real, not sidelobe effect;

1-70.-H I emission on edge of bandpass, poor sensitivity, parameters uncertain;

1-98. - Very near edge of bandpass; poor sensitivity, parameters uncertain;

1-109.-Alternative optical identification: $120521.3+153110$, a much fainter object but nearer to the $\mathrm{H}$ i position;

1-113. - 120625.3+132303 is in a pair with $120626.0+132254$; $\mathrm{H}$ I emission could be associated with either or both;

1-116. - No optical counterpart, extended H I; compact HVC (cHVC)?

1-122.-No optical counterpart, extended H I; HVC;

1-124. - H I emission on edge of bandpass, poor sensitivity, parameters uncertain;

1-129.- - H m merges in spectral region affected by RFI; parameters of detection very uncertain;

1-130. - No optical counterpart; extended H i; HVC projected in vicinity of NGC 4192;

1-148. - No optical counterpart; extended H I; HVC projected in vicinity of NGC 4192;

1-153. - No optical counterpart; extended H i; HVC projected in vicinity of NGC 4192;

1-157.- No optical counterpart; extended H I; HVC projected in vicinity of NGC 4192;

1-158.-H I emission may have southwest-to-northeast extension;

1-162.-No optical counterpart; extended H I; HVC projected in vicinity of NGC 4192;

1-173.-No identifiable optical counterpart;

1-184. - No identifiable optical counterpart; extended H I; HVC projected in vicinity of NGC 4192;

1-185. - No identifiable optical counterpart;

1-210. - No optical counterpart, south of UGC 7284; galaxy appendage or HVC?

1-214.- - H i position matches that of IC 3080, which has discordant optical $z$; match with $121607.0+141237$ based on similarity of $z$, but note large positional discrepancy; ambiguous optical identification; 
TABLE 1

H i Candidate Detections

\begin{tabular}{|c|c|c|c|c|c|c|c|c|c|c|c|c|c|}
\hline \multirow[b]{2}{*}{$\begin{array}{c}\text { Catalog Number } \\
\text { (1) }\end{array}$} & \multirow[b]{2}{*}{$\begin{array}{l}\mathrm{AGC} \\
(2)\end{array}$} & \multicolumn{2}{|c|}{ H I Coordinates (J2000.0) } & \multicolumn{2}{|c|}{ Optical Coordinates (J2000.0) } & \multirow{2}{*}{$\begin{array}{c}c z_{\odot} \\
\left(\mathrm{km} \mathrm{s}^{-1}\right) \\
(7)\end{array}$} & \multirow{2}{*}{$\begin{array}{c}W 50\left(\epsilon_{w}\right) \\
\left(\mathrm{km} \mathrm{s}^{-1}\right) \\
(8)\end{array}$} & \multirow{2}{*}{$\begin{array}{c}F_{c}\left(\epsilon_{f}\right) \\
\left(\mathrm{Jy} \mathrm{km} \mathrm{s}^{-1}\right) \\
(9)\end{array}$} & \multirow[b]{2}{*}{$\begin{array}{l}\mathrm{S} / \mathrm{N} \\
(10)\end{array}$} & \multirow{2}{*}{$\begin{array}{c}\mathrm{rms} \\
(\mathrm{mJy}) \\
(11)\end{array}$} & \multirow{2}{*}{$\begin{array}{c}\text { Distance } \\
(\mathrm{Mpc}) \\
(12)\end{array}$} & \multirow{2}{*}{$\begin{array}{c}\log M_{\mathrm{HI}} \\
\left(M_{\odot}\right) \\
(13)\end{array}$} & \multirow[b]{2}{*}{$\begin{array}{l}\text { Code } \\
(14)\end{array}$} \\
\hline & & $\begin{array}{l}\text { R.A. } \\
\text { (3) }\end{array}$ & $\begin{array}{l}\text { Decl. } \\
\text { (4) }\end{array}$ & $\begin{array}{l}\text { R.A. } \\
(5)\end{array}$ & $\begin{array}{l}\text { Decl. } \\
(6)\end{array}$ & & & & & & & & \\
\hline $1-1$ & 210692 & 114419.8 & +150620 & 114420.2 & +150616 & 10489 & $283(13)$ & $1.42(0.08)$ & 8.6 & 1.85 & 151.5 & 9.89 & 1 \\
\hline $1-2 \ldots \ldots \ldots \ldots \ldots \ldots$ & 215418 & 114421.4 & +150459 & & & 247 & $33(2)$ & $0.38(0.04)$ & 6.8 & 2.09 & $\ldots$ & $\ldots$ & $9^{*}$ \\
\hline $1-3$ & 213528 & 114432.1 & +131527 & 114432.0 & +131511 & 10291 & $119(12)$ & $0.52(0.06)$ & 4.8 & 2.09 & 148.6 & 9.43 & 2 \\
\hline 1-4 …….................... & 213473 & 114437.1 & +124657 & 114439.5 & +124726 & 12751 & $245(88)$ & $1.06(0.09)$ & 5.8 & 2.14 & 183.7 & 9.93 & 2 \\
\hline $1-5 \ldots \ldots \ldots \ldots \ldots \ldots \ldots$ & 212928 & 114440.6 & +144551 & 114440.9 & +144555 & 10240 & $379(4)$ & $2.32(0.08)$ & 14.0 & 1.73 & 147.9 & 10.08 & 1 \\
\hline $1-6 \ldots \ldots \ldots \ldots \ldots \ldots \ldots \ldots$ & 215197 & 114442.2 & +150215 & 114444.0 & +150140 & 3354 & $103(40)$ & $0.72(0.05)$ & 7.1 & 1.78 & 50.4 & 8.63 & 1 \\
\hline $1-7 \ldots \ldots \ldots \ldots \ldots \ldots \ldots \ldots$ & 215148 & 114443.7 & +121708 & 114442.6 & +121802 & 10258 & $303(76)$ & $1.20(0.09)$ & 6.5 & 2.11 & 148.1 & 9.79 & 1 \\
\hline $1-8$ & 210746 & 114524.6 & +142242 & 114522.1 & +142200 & 3342 & $129(25)$ & $0.90(0.05)$ & 10.8 & 1.56 & 50.3 & 8.73 & 1 \\
\hline $1-9$ & 210753 & 114533.5 & +121240 & 114534.9 & +121218 & 9298 & $92(3)$ & $1.40(0.06)$ & 14.4 & 2.05 & 134.5 & 9.78 & 1 \\
\hline $1-10$ & 215149 & 114554.6 & +134955 & 114556.1 & +135021 & 3267 & $38(9)$ & $0.57(0.04)$ & 7.6 & 1.77 & 49.2 & 8.51 & 1 \\
\hline
\end{tabular}

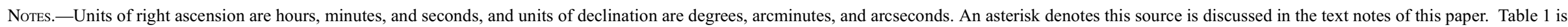
published in its entirety in the electronic edition of the Astronomical Journal. A portion is shown here for guidance regarding its form and content. 
1-217.-No identifiable optical counterpart; extended H I; HVC projected in vicinity of NGC 4192;

1-228. - No identifiable optical counterpart; extended H I; HVC projected in vicinity of NGC 4192;

1-229.-Alternative optical identification is $121716.7+142732$; optical brighter but farther from $\mathrm{H}$ i center;

1-235. - No identifiable optical counterpart; portion of Virgo HI21 (Davies et al. 2004; Minchin et al. 2005);

1-238. - No identifiable optical counterpart; portion of Virgo HI21 (Davies et al. 2004; Minchin et al. 2005);

1-239.- No identifiable optical counterpart; portion of Virgo HI21 (Davies et al. 2004; Minchin et al. 2005);

1-241.- - In vicinity of Virgo HI21 (Davies et al. 2004; Minchin et al. 2005);

1-242.-No identifiable optical counterpart; unresolved, compact HVC projected in vicinity of NGC 4192;

1-245.-No identifiable optical counterpart; unresolved, compact HVC projected in vicinity of NGC 4192;

1-257. - No identifiable optical counterpart; $4^{\prime}$ north of H I $121910.9+125322$ (=AGC 220351); tidal appendage?

1-260.-Resolved disk, elongated southwest (high velocity) to northeast (low velocity); previous $\mathrm{H} \mathrm{I} c z=1280$ appears to be wrong;

1-263. - Crowded optical field, ambiguous optical identification; alternative optical counterparts are 121942.5+132549, 121948.4+132522, and 121945.1+132627;

1-264.-No identifiable optical counterpart; barely resolved, compact HVC;

1-271.-No identifiable optical counterpart; VCC 429 at $122043.8+143751$ and similar $z$ is at $2.3^{\prime}$ to northeast; $\mathrm{H}$ I assumed related;

1-276.-Blend of UGC 7412 and UGC 7418 (NGC 4298 and NGC 4302); H I flux mainly of UGC 7418;

1-278. - Optical counterpart is a close galaxy pair;

1-279.-No identifiable optical counterpart; unresolved, compact HVC;

1-287.-Very uncertain separation from Milky Way H i; marginal detection and very poor $\mathrm{H}$ i parameters;

1-309. - Uncertain separation from Milky Way H I;

1-312.-Very poor positional match, marginal $\mathrm{S} / \mathrm{N}$ and identification;

1-316.-Located 3.6' to southeast of M86 (UGC 7532), no identifiable optical counterpart; part of Virgo HI4 (Davies et al. 2004; note that the declination listed in that paper is incorrect) and shown to be a plume extending from NGC 4388 by Oosterloo \& van Gorkom (2005);

1-318.-Uncertain separation from Milky Way H I;

1-336. - Extended H I source, blend of $123115.0+141148$ and 123120.0+131144; identification assignment of $\mathrm{H}$ i to $123115.0+$ 141148 on proximity grounds;

1-347. - Fainter optical galaxy at $123355.2+135554$ also a possible counterpart;

1-352.-No clearly identifiable optical counterpart;

1-354. - No identifiable optical counterpart; in spite of fair $\mathrm{S} / \mathrm{N}$, doubts on reality, due to standing waves produced by proximity to M87;

1-359.-Optical counterpart is extremely LSB, very extended object? However, the proximity of M87 makes H I detection somewhat doubtful;

1-360.-No identifiable optical counterpart, marginal S/N;

1-366. - No identifiable optical counterpart;

1-371.-Ambiguous optical identification; $123650.8+141506$ possible; AGC 224865, identified with $123643.1+141611,2.7^{\prime}$ to northwest, is at similar $z$ (NGC 4571 in foreground);
1-377.-Optical identification ambiguous; other possibilities are $123857.5+142435$ and $123859.1+142457$;

1-386. - On edge of band, ragged data;

1-391.-Blend with 7874 (NGC 4633), interacting system, and interference with Milky Way $\mathrm{H}$ I; parameters uncertain;

1-392.-Most emission in profile associated with source UGC 7874, identified with UGC 7884 (NGC 4639) 2.8' east; feature measured is wing to high-velocity side of emission peak;

1-397.-Extended emission of UGC 7902 (NGC 4654) overwhelms the field; emission tentatively assigned to optical galaxy at $124412.0+125631$ is the low-velocity wing of line;

1-398.-Positional offset of $1^{\prime}$ between optical and $\mathrm{H}$ i probably real, not centroiding error;

1-399. - Optical identification with very faint LSB feature, 3.5' to southeast of IC 3720 ;

1-402.-Ambiguous optical ID: also possible 124514.7+141906;

1-409. - On edge of band, ragged data;

1-417. - Optical identification with faint blue object is very tentative;

1-426.-Other possible optical counterpart at 125209.6+150456, marginally farther away;

1-451.-Alternative optical identification: fainter object at $125911.0+142519$;

1-474.-Optical identification with bluest galaxy in triplet;

1-488. - On edge of band, ragged data;

1-493. - On edge of band, ragged data;

1-494.-No identifiable optical counterpart, extended H I; part of HVC complex;

1-497.-No identifiable optical counterpart, extended H I; part of HVC complex;

1-500.- No identifiable optical counterpart, extended $\mathrm{H}$.; part of HVC complex;

1-502.- No identifiable optical counterpart, extended H I; part of HVC complex;

1-504. - Galactic emission merges in region affected by RFI; parameters uncertain;

1-507.-Galactic emission merges in region affected by RFI; parameters uncertain;

1-513.-No identifiable optical counterpart, extended H I; part of HVC complex;

1-524. - Galactic emission merges in region affected by RFI: parameters uncertain;

1-531. - On edge of region affected by RFI; identification with very faint, LSB object is tentative; caveat emptor;

1-532.-Galactic emission merges in region affected by RFI; parameters uncertain;

1-534. - On edge of band, ragged data;

1-538. - Galactic emission merges in region affected by RFI; parameters uncertain;

1-541. - Galactic emission merges in region affected by RFI; parameters uncertain;

1-543. - Galactic emission merges in region affected by RFI; parameters very uncertain;

1-556. - No identifiable optical counterpart; compact HVC;

1-563. - Galactic emission merges in region affected by RFI, but parameters only mildly affected;

1-578. - No unambiguous optical counterpart; blue object in vicinity; marginal detection;

1-611.-NGC 5221, highly disturbed;

1-613.-Optical identification assigned to blue object superposed onto E galaxy at similar $c z$; merger underway? NGC 5221 is $6^{\prime}$ north, highly disturbed;

1-621.- - On edge of band, ragged data; 
1-636.-Ambiguous optical identification; possible optical counterparts are $134124.7+151630$ and $134119.4+151553$;

1-639.-H i source may be extended to northwest and southeast of center;

1-645.-Ambiguous optical identification; 134233.9+130210 is an alternative candidate, $20^{\prime \prime}$ farther from $\mathrm{H}$ i center;

1-665.-Marginal detection; tight pair; other possible optical counterpart at $134739.2+154404$;

1-667.-Blend; optical counterpart is interacting pair VII Zw 338;

1-673.-Ambiguous optical identification; 134941.6+155702 is an alternative candidate;

1-692.-Extended H I, no optical counterpart; HVC;

1-700.-H I emission merges in RFI; parameters highly uncertain; optical galaxy in pair, alternative possible optical counterpart at $135452.3+140741$;

1-710.-Emission blended with AGC 233714, parameters uncertain;

1-711.-See AGC 230859;

1-722. - Blend with emission by several other objects within $1.5^{\prime}$

\section{STATISTICAL PROPERTIES OF THE ALFALFA H I SAMPLE}

The distribution by detection code of $\mathrm{H}$ I detections listed in Table 1 is as follows: 24 (4\%) are HVCs, 120 (16\%) are of type 2, i.e., priors of low $\mathrm{S} / \mathrm{N}$ but corroborated by optical redshifts, and $586(\sim 80 \%)$ are detections of type 1 , of fair to excellent quality.

The $132 \mathrm{deg}^{2}$ region of the sky covered by the sample of $\mathrm{H}$ I detections listed in Table 1 has been intensely investigated by a variety of studies, including surveys of the Virgo Cluster and the SDSS. Targeted observations of optically selected samples amounting to many hundreds of hours of telescope time have yielded detections for 158 objects in the Galaxy On Line Database Milano Network compilation of Gavazzi et al. (2003), some of which are fainter than the ALFALFA detection limit (and required a lot more integration time). The blind H I Virgo HIJASS survey (Davies et al. 2004) is partly contained within the survey area presented here. In the HIJASS region of full sensitivity which overlaps our ALFALFA data set, HIJASS found $15 \mathrm{H}$ i detections; in contrast, we detect $193 \mathrm{H}$ I sources in the same region. We detect both the H i plume found near NGC 4388 (Oosterloo \& van Gorkom 2005), referred to as Virgo HI4 by Davies et al. (2004), and the extended emission in the vicinity of the "dark galaxy" Virgo HI21 (Davies et al. 2004; Minchin et al. 2005) showing a clear connection to NGC 4254; we will discuss this structure elsewhere (M. P. Haynes et al. 2007, in preparation). Likewise, the improvement over HIPASS is impressive. In the region containing the $730 \mathrm{H}$ I detections included in Table 1, the HIPASS data set includes only 40 objects (Wong et al. 2006). Of those, several show large position offsets with respect to multiple ALFALFA detections lying within a single Parkes beam, and two are not confirmed by ALFALFA.

The median distance of this sample is $102 \mathrm{Mpc}$. As of late August of $2006,72 \%$ of the ALFALFA H I detections presented here have optical or $\mathrm{H}$ I redshifts previously known, and $28 \%$ are new. The percentage of ALFALFA sources that are new $\mathrm{H}$ I detections is $69 \%$. In view of the fact that numerous $\mathrm{H}$ i studies have been conducted in this region, largely based on optically selected targets, the percentage of $69 \%$ new $\mathrm{H}$ I detections illustrates the fact that previous $\mathrm{H}$ i surveys, generally based on fluxor size-limited, optically selected samples, missed the majority of H I sources; the conventional wisdom on which optical targets would turn out to be $\mathrm{H}$ I-rich appears to have been limited.
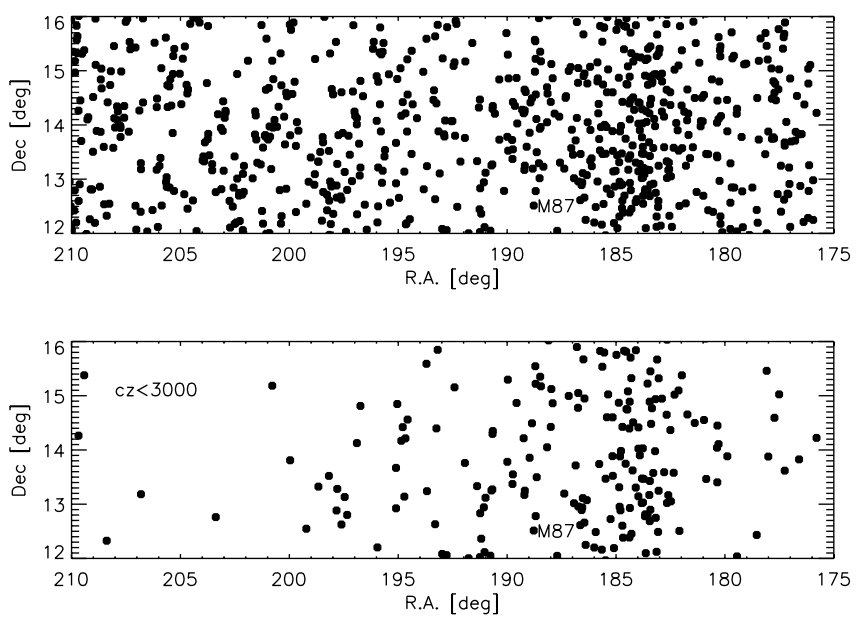

FIG. 3.-Sky distribution of H I candidate detections listed in Table 1: top, all objects; bottom, objects with $c z<3000 \mathrm{~km} \mathrm{~s}^{-1}$. A label identifies the position of M87, in the vicinity of which H I sources cannot be effectively surveyed because of standing waves and the large increase in system temperature contributed by its strong continuum emission; see $\S 2.2$.

About $25 \%$ of ALFALFA detections have $c z<3000 \mathrm{~km} \mathrm{~s}^{-1}$. The fraction of local objects in the catalog presented here is enhanced by the fact that the region sampled crosses the supergalactic plane and the northern part of the Virgo Cluster, one of the densest regions of the Local Supercluster. The detection rate, of about 5.4 objects ( 4.4 of detection code 1 ) per square degree, is thus enhanced by a circumstance associated with the characteristics of the large-scale structure of the galaxy distribution in the local universe. Were it not for the enhancement in the detections within the Local Supercluster, the detection rate would have been lower by about one-fifth. This can be visually gauged by inspection of Figure 3, which shows the sky distribution of all the detected sources in the top panel and that of objects with $c z<3000 \mathrm{~km} \mathrm{~s}^{-1}$ in the bottom panel.

As mentioned in $\S 2.2$, the region of about $1^{\circ}-1.5^{\circ}$ radius about the location of M87 is contaminated by the high brightness of its strong radio source, which not only increases the system temperature but also induces strong standing waves. Therefore, the sky region around M87 cannot be sampled by H i spectroscopy, except for the very brightest $\mathrm{H}$ I sources. The location of M87 is indicated in Figure 3, at the center of a region of rarefied density of $\mathrm{H}$ I detections.

Figure 4 (top) shows the redshift distribution of the galaxies in the current sample. The signatures of the Virgo Cluster at the low-velocity end and of other features in the large-scale structure of the local universe are clearly evident. The lack of detections on a strip nearly $1000 \mathrm{~km} \mathrm{~s}^{-1}$ wide near $c z \simeq 15,000 \mathrm{~km} \mathrm{~s}^{-1}$ reflects a bias of the survey. It corresponds to a spectral region heavily and nearly continuously affected by RFI, as shown in Figure 1. Figure 4 (bottom) shows the analogous diagram for the galaxies with known optical redshifts in the same region of the sky. The large-scale distribution properties of both samples are very similar. The most notable difference between the two plots is the rarefaction of sources at higher $c z$ in the $\mathrm{H}$ i panel.

Figure 5 summarizes the distribution in the values of heliocentric velocity $c z_{\odot}$, velocity width $W 50$, flux integral $F_{c}, \mathrm{~S} / \mathrm{N}$, and $\mathrm{H}$ I mass $M_{\mathrm{H} \text { I }}$ for the candidate $\mathrm{H}$ I detections reported in this catalog. We remind the reader that this survey is not designed to be complete to a given flux integral limit, but rather that the sensitivity limit is a function of velocity width, as discussed in Paper I, Paper II, and further below. We also note the paucity of sources 

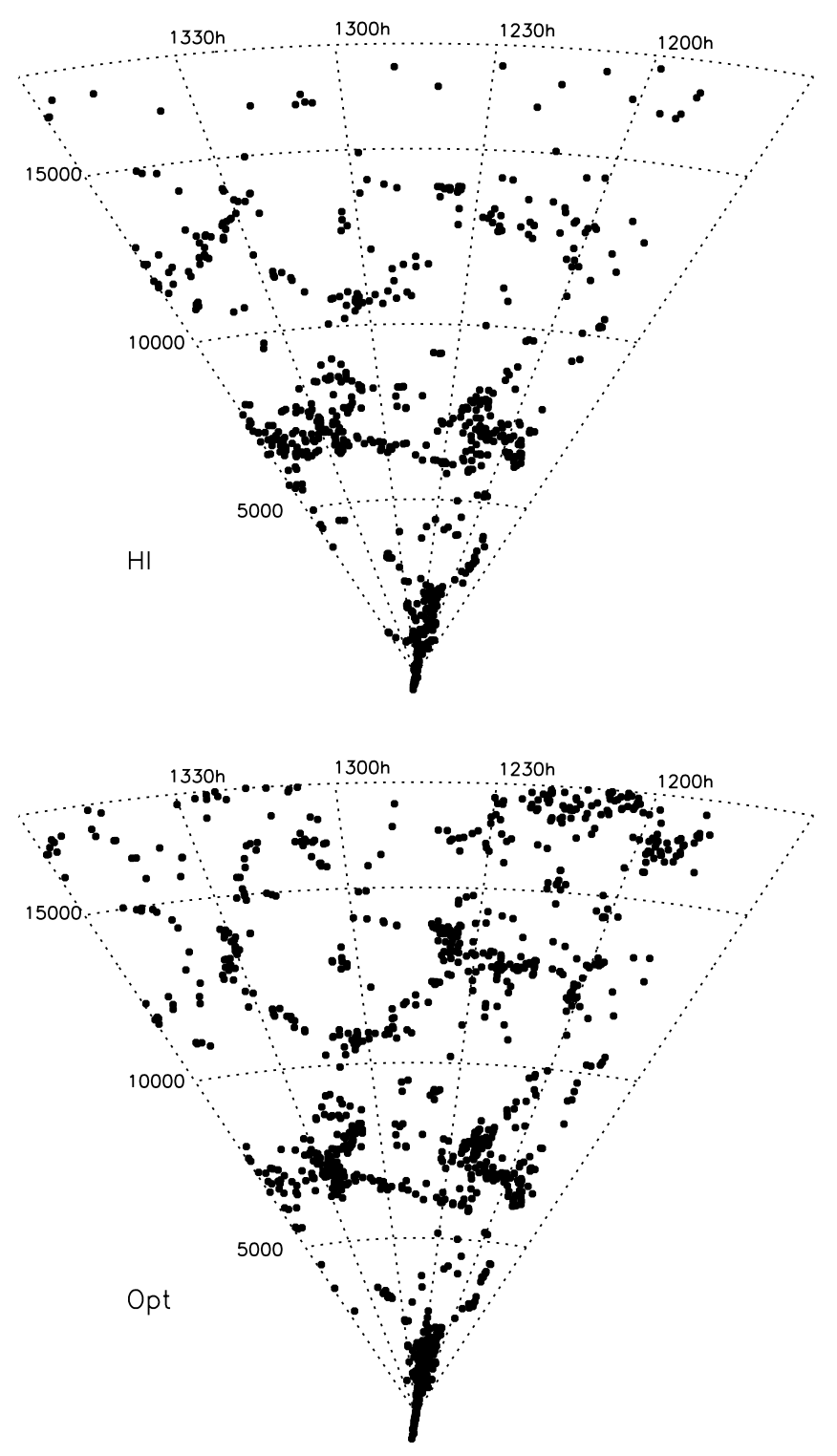

FIG. 4.- Right ascension vs. recession velocity in $\mathrm{km} \mathrm{s}^{-1}$, of all sources listed in Table 1 (top; $\mathrm{H} \mathrm{I}$ ), and the analogous plot of galaxies with optical redshifts in the same region of sky (bottom; Opt). Note the Virgo Cluster in the lower portion of each cone plot.

with reported $\mathrm{H}$ I mass below $10^{7} M_{\odot}$ in Figure $5 e$; while this may be due to a truly low cosmological population of low-mass $\mathrm{H}$ I sources, the effect is likely to be, at least in part, spurious. As distances have been inferred from redshifts alone, albeit with the aid of a peculiar velocity flow model, the model yields highly uncertain distances within the region sampled, due to the presence of the Virgo Cluster; a very nearby, low velocity object is far more likely to be assigned the Virgo Cluster distance by the flow model than a Virgo Cluster object of low velocity to be assigned a low distance. The presence of the Virgo Cluster may thus operationally "displace" nearby objects, if any, making them appear farther than they truly are. Figure $5 e$ suggests that such may indeed be the case, as the number of objects plotted between $10^{7.5}$ and $10^{8} M_{\odot}$ is overabundant for any reasonable shape of the $\mathrm{H}$ i mass function, in comparison with the higher mass bins. Redshift-independent distance estimates of those objects are necessary, if a reliable determination of the $\mathrm{H}$ I mass function in Virgo and in the field is to be obtained. This issue will be addressed in a forthcoming paper by B. R. Kent et al. (2007, in preparation).
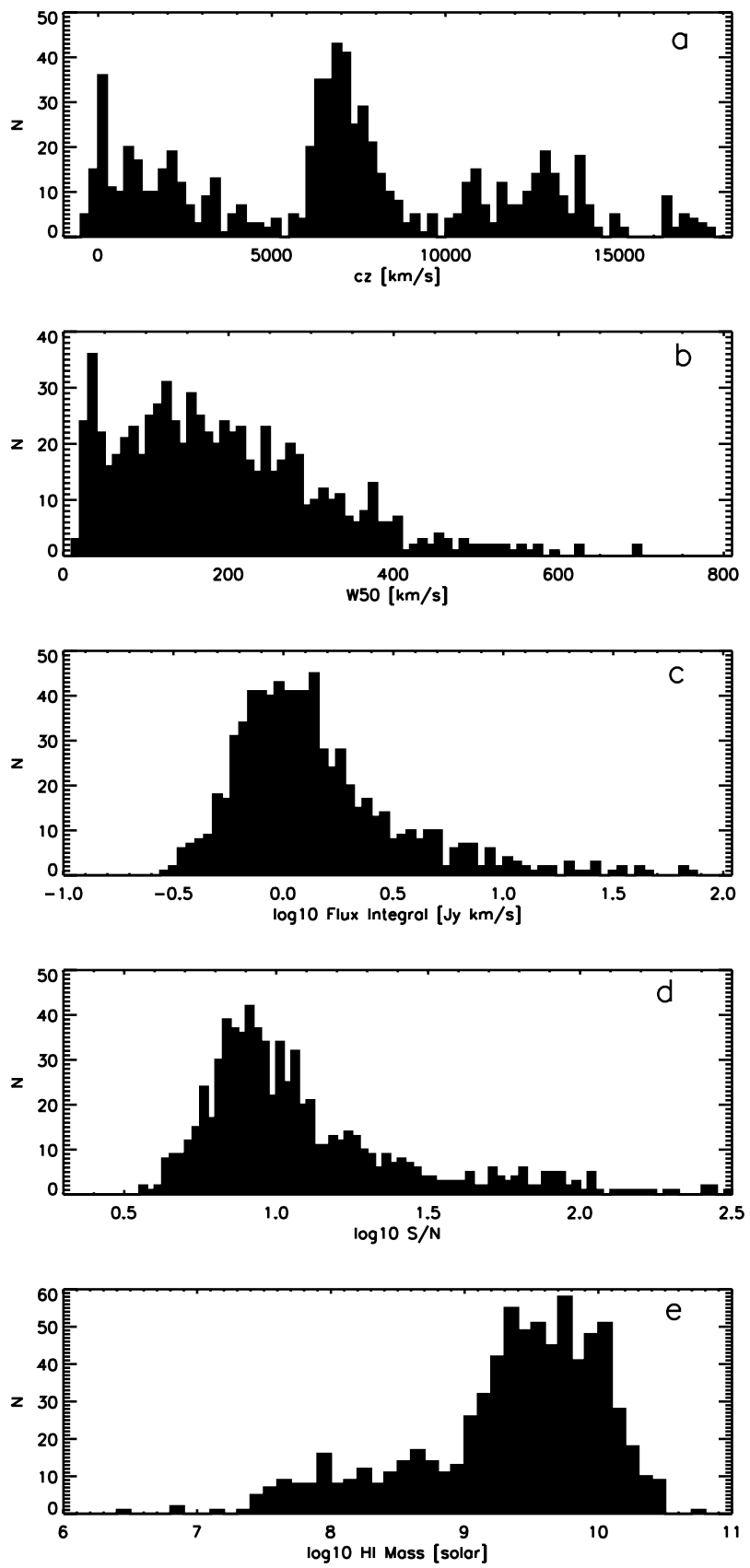

FIG. 5. - Histograms of the $\mathrm{H}$ I candidate detections listed in Table 1. From top to bottom: (a) recessional velocity in $\mathrm{km} \mathrm{s}^{-1} ;\left(\right.$ b) $\mathrm{H}$ I line width $W 50$ in $\mathrm{km} \mathrm{s}^{-1}$; (c) $\log$ base 10 of the flux integral in Jy km s${ }^{-1} ;(d)$ the peak $\mathrm{S} / \mathrm{N}$, in logarithmic units; (e) the derived H I mass, in logarithmic units of $M_{\odot}$. Note the "step" in the $\mathrm{H}$ I mass histogram near $10^{7.5}$, which is probably an artifact due to uncertainties in galaxy distances in the direction toward the Virgo Cluster, as described in the text.

Figure $6 a$ displays a plot of $\mathrm{H}$ I mass versus distance for all objects in Table 1. Vertical dotted lines outline the distance interval within which our survey is unable to detect any cosmic sources due to the intrusion of RFI. Galaxies in the Virgo Cluster appear as the vertical feature near $D=17 \mathrm{Mpc}$. Figure $6 b$ shows that the $\mathrm{S} / \mathrm{N}$ limit of the survey is relatively independent of velocity width. Sources with velocity widths larger than a few hundred $\mathrm{km} \mathrm{s}^{-1}$ are apparently identified reliably by our signal-extraction algorithm. Very few objects are found with velocity width smaller than $25 \mathrm{~km} \mathrm{~s}^{-1}$. Several candidate detections are obtained with narrow widths; they lie generally near the detection limit and have 

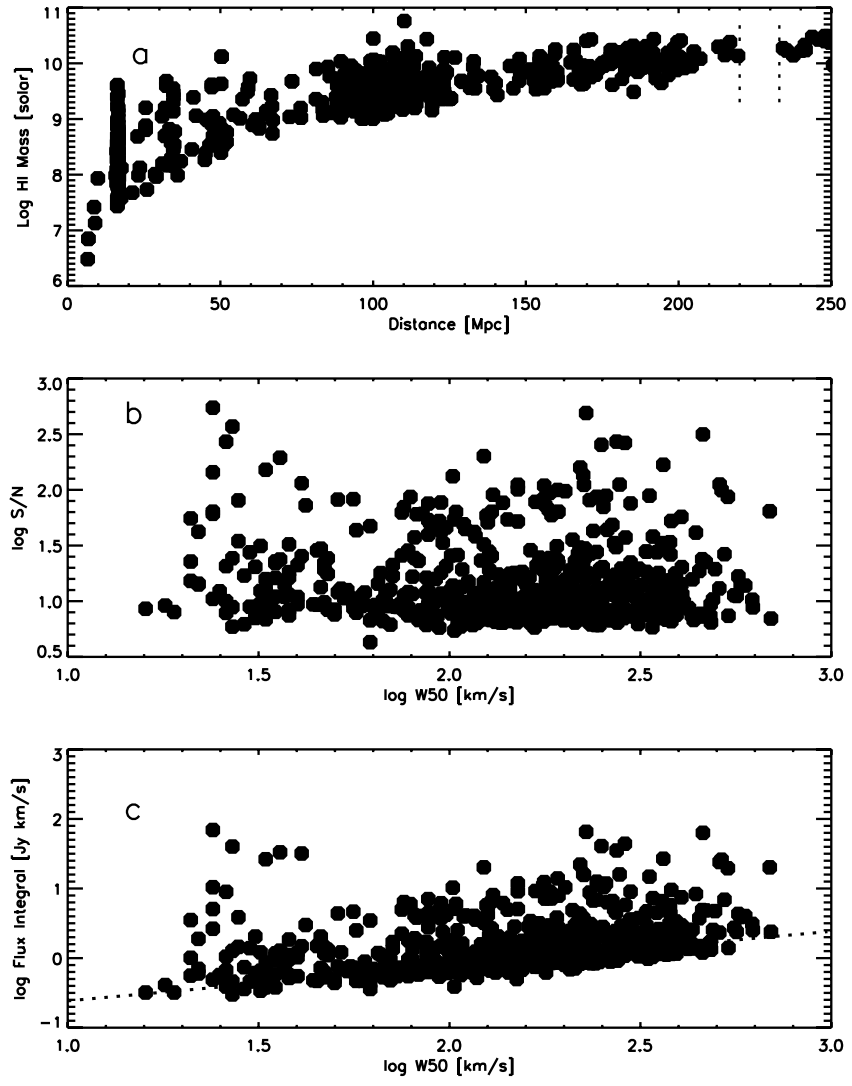

FIG. 6.- (a) H I mass vs. distance for all sources of types 1 and 2 in Table 1 The Virgo Cluster is the vertical feature near $D=17 \mathrm{Mpc}$. Cosmic sources within the spectral region between the two vertical dashed lines are inaccessible due to RFI. (b) S/N vs. velocity width for all sources in Table 1 . The lower envelope appears to be independent of $\mathrm{S} / \mathrm{N}$, indicating that no significant bias is present in the detection of sources of large width. (c) Flux integral vs. velocity width. The dashed line corresponds to a $\mathrm{S} / \mathrm{N}$ limit of 6.5 .

no identifiable optical counterparts. Most of them are believed to be due to RFI. Determination of their nature will require follow-up corroborating observations.

Figure $6 c$ shows the dependence of the survey's limiting flux integral on velocity width. The dotted line indicates a detection threshold of $\mathrm{S} / \mathrm{N}=6.5$, assuming that a spectral smoothing width of $W 50 / 20$ can be adopted for features as wide as $400 \mathrm{~km} \mathrm{~s}^{-1}$, and a constant value of $400 / 20$ for features wider than $400 \mathrm{~km} \mathrm{~s}^{-1}$ (see description of col. [10] of Table 1). The fact that the lower envelope of the data points appears consistent with a slope of $1 / 2$, rather than steepening to a slope of 1 , indicates that our adoption of a smoothing width is essentially correct.

\section{POSITIONAL ACCURACY OF H i SOURCES}

The main purpose of this work is to make available to the general community a data set of $\mathrm{H}$ I detections, with analysis of the overall properties of the sample kept at a minimum in the interest of speedy delivery. We address the reader to Paper I and Paper II, in which a preliminary analysis was discussed. However, because of the dependence of the performance properties of the Arecibo telescope on the direction in which it points, some characteristics of the ALFALFA samples will be sky-zone dependent. One of such characteristics is the positional accuracy of the $\mathrm{H}$ I detections, which carry the imprint of the telescope pointing errors. Thus, we devote this section to a brief analysis of that matter. The corrections we find are specific to the data set presented here.
Positional accuracy of $\mathrm{H}$ i sources is of paramount importance per se and in making identification with sources from other catalogs. The main limiting factor for ALFALFA sources is of course the resolution of the ALFA beam. As discussed in Paper I, the ALFA beams are slightly elliptical, with half power full-widths of $3.3^{\prime}$ and $3.8^{\prime}$. The major axis of the beam is always directed along the position of the telescope's azimuth arm; the vast majority of the ALFALFA observations and all those presented in this paper are made with the azimuth arm in the north-south direction; thus, the beam's major axis is in the declination direction.

ALFALFA samples the sky every second in R.A. and every arcminute in declination. However, sources are extracted and their positions measured after the data are converted to a spatial grid sampled at $1^{\prime} \times 1^{\prime}$. A Gaussian weight function is applied as part of the regridding process, which reduces the spatial resolution of the data to $3.8^{\prime} \times 4.3^{\prime}$. An automatic source-extraction algorithm identifies a source candidate, which is successively measured and extracted interactively by an observer. The spectral extent of the feature is gauged and a two-dimensional map of the emission is integrated over that full spectral extent. Ellipses are fitted to the image at a set of fixed isophotal levels, as well as at the half-power and quarter-power of the peak level, as measured in flux density units per beam area. The position of the source is assumed, in all cases, to be the center of the half-power ellipse. This has proved to be the best choice for the vast majority of sources, as they are generally unresolved by the ALFA beam. Caution is necessary when sources are extended and do not display a clear center of symmetry. A more detailed description of the positional parameter estimates will be presented elsewhere, together with the overall description of the ALFALFA dataprocessing pipeline (R. Giovanelli et al. 2007, in preparation).

The second most important parameter regulating the quality of the ALFALFA positions is the $\mathrm{S} / \mathrm{N}$ of the $\mathrm{H}$ I emission. High $\mathrm{S} / \mathrm{N}$ sources allow more accurate centroiding than low $\mathrm{S} / \mathrm{N}$ ones, as discussed below.

The third important influence on ALFALFA positions is the quality of the Arecibo telescope pointing. The telescope can set with a repeatable accuracy of a few arcseconds; however, the pointing algorithms which use fits to the telescope configurational parameters yield systematic pointing errors which may, at the L band, add up to $15^{\prime \prime}$ or more. The highest pointing errors occur at the lowest zenith angles. The chosen observing mode for ALFALFA, which freezes the telescope configuration at fixed azimuth, largely allows recovery and correction of the systematic pointing errors.

Positional accuracy is discussed here by using the positional differences between the $\mathrm{H}$ I emission ellipse centers and the centers of the galaxies which are identified as optical counterparts. Those differences will include systematic telescope pointing offsets, occasional mismatch in the extent and center of optical and $\mathrm{H}$ i source, statistical errors in the $\mathrm{H}$ I centroiding and misidentification of optical counterparts. Errors in the centroiding of the optical sources are negligible in comparison. A similar exercise to the one described below has been carried out by comparing ALFALFA positions of continuum radio sources with interferometic positions. Those results are in agreement with those discussed here and will be presented elsewhere (B. R. Kent et al. 2007, in preparation).

Figure 7 shows the positional differences ( $\mathrm{H}$ I minus optical) in R.A. ( $\Delta \mathrm{ra})$ and in declination $(\Delta \mathrm{dec})$, respectively, expressed in arcseconds. The data are split into four panels differing in declination range, within bins of $1^{\circ}$. The frame labeled "Dec 12" refers to sources between decl. $=+12^{\circ}$ and $+13^{\circ}$, and so on. As the observations were made with the telescope's azimuth arm 

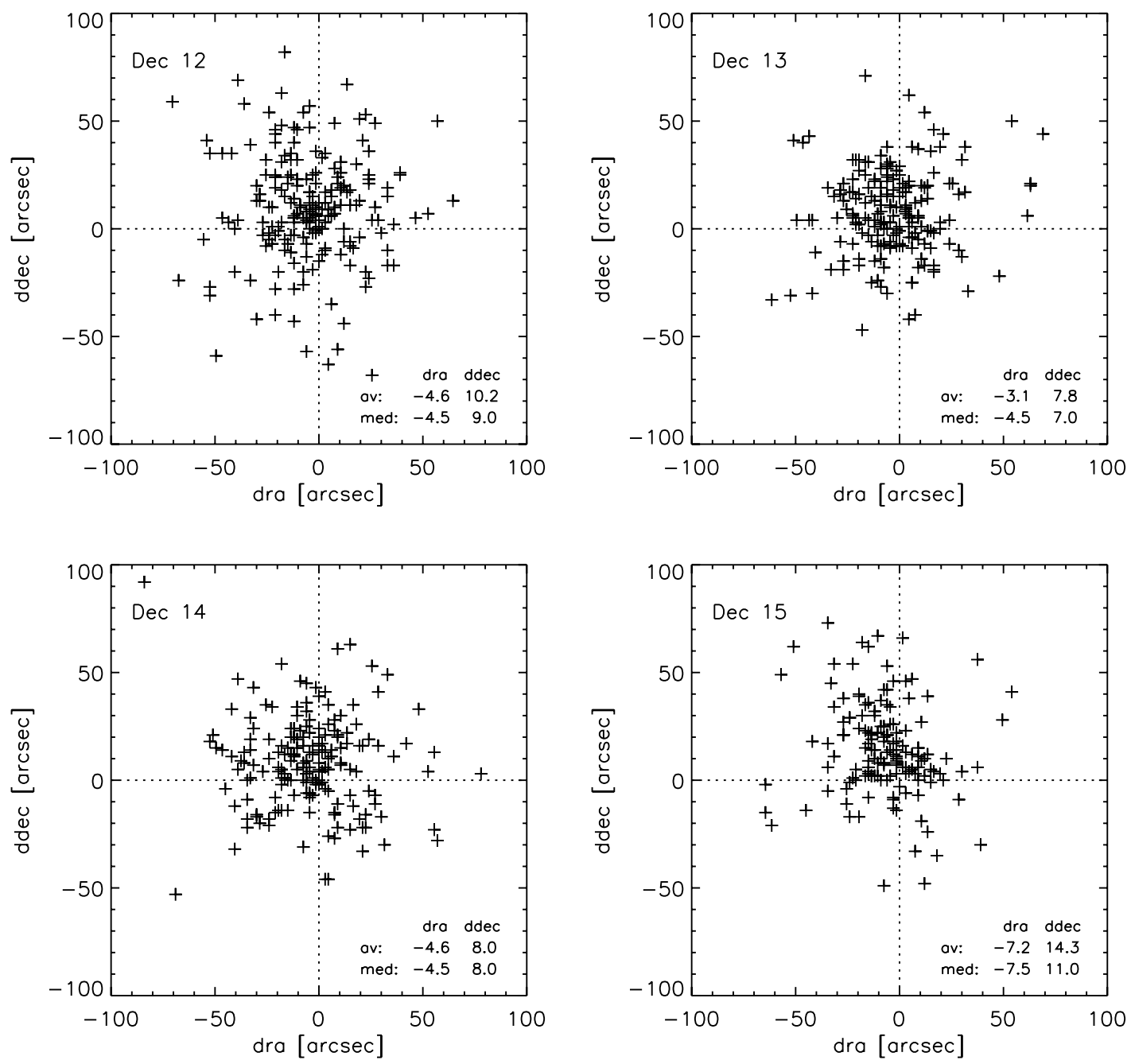

FIG. 7.-Differences between the positions of the $\mathrm{H}$ I sources, before applying a correction for telescope pointing errors, and the optical counterpart positions as listed in cols. (5) and (6) of Table 1. Sources are separated by declination bins of $1^{\circ}$. In each panel, the label in the top left corner (e.g., "Dec 12 ") identifies the declination of the sources (e.g., between $+12^{\circ}$ and $+13^{\circ}$ for Dec 12). Average and median offsets, expressed in arcseconds, are inset within each panel.

oriented north-south, a degree of declination converts exactly in $1^{\circ}$ of zenith angle; the latitude of the Arecibo telescope is $+18^{\circ} 20^{\prime} 37^{\prime \prime}$ north. The plot includes all the sources presented in Table 1 for which an optical identification was made. The H I positions used are those obtained before any correction for telescope pointing errors was applied. These sources were all observed at the same azimuth of $0^{\circ}$ and zenith angles between $2.3^{\circ}$ and $6.4^{\circ}$. A systematic offset in the center of the distribution toward positive $\Delta \mathrm{ra}$ and negative $\Delta \mathrm{dec}$ is apparent in all panels, with that offset becoming progressively larger with increasing declination, i.e., decreasing zenith angle. This offset mimics the telescope pointing errors, ${ }^{19}$ which increase as the telescope points closer to the site's zenith. The amplitude of the pointing errors is indicated by the insets in each plot, expressed respectively by the mean and the median.

Figure 8 shows the distribution of positional offset between $\mathrm{H}$ I and optical positions after the systematic pointing errors described above have been removed from the $\mathrm{H}$ i positions. The separation of the data into four panels in this case is by $\mathrm{S} / \mathrm{N}$. The systematic pointing error that was removed is a simple function of declination, i.e., zenith angle, obtained for each source by inter-

\footnotetext{
${ }^{19}$ See "Pointing errors" at http://www.naic.edu/ phil/sysperf/sysperf.html\#alfa.
}

polating between the offsets shown in Figure 7. The H I source positions corrected for this systematic error are those listed in column (2) of Table 1. The dispersion of the positional differences about the center is, as expected, dependent on $\mathrm{S} / \mathrm{N}$. For the higher $\mathrm{S} / \mathrm{N}$ objects $(\mathrm{S} / \mathrm{N}>12)$, ALFALFA positions are on average accurate to about $15^{\prime \prime}$. ALFALFA positions are significantly better than those obtained with the precursor run reported by Paper II (median difference between $\mathrm{H}$ I and optical position of $34^{\prime \prime}$ ). This is due to two reasons: (1) the ALFALFA data include two full sweeps of each region of the sky with ALFA, hence yielding twice as dense a spatial sampling in declination as most of the data in the precursor run, and (2) the technique used to extract positional information, which fits ellipses to isophotal contours, is more accurate than that used for the precursor run data.

\section{NEW DISCOVERIES, FUTURE WORK, AND CONCLUSIONS}

The principal aim of ALFALFA is to obtain an accurate census of $\mathrm{H}$ I-bearing objects in the local universe. The catalog of candidate $\mathrm{H}$ I detections presented in Table 1 yields a first picture of the "ALFALFA sky." As discussed in $\S 4$ and shown in Figure 4, ALFALFA detections span 5 orders of magnitude in $\mathrm{H}$ I mass, and include both massive spirals out to $z \sim 0.06$ and dwarf galaxies within a few megaparcsecs of the Milky Way. The region sampled 

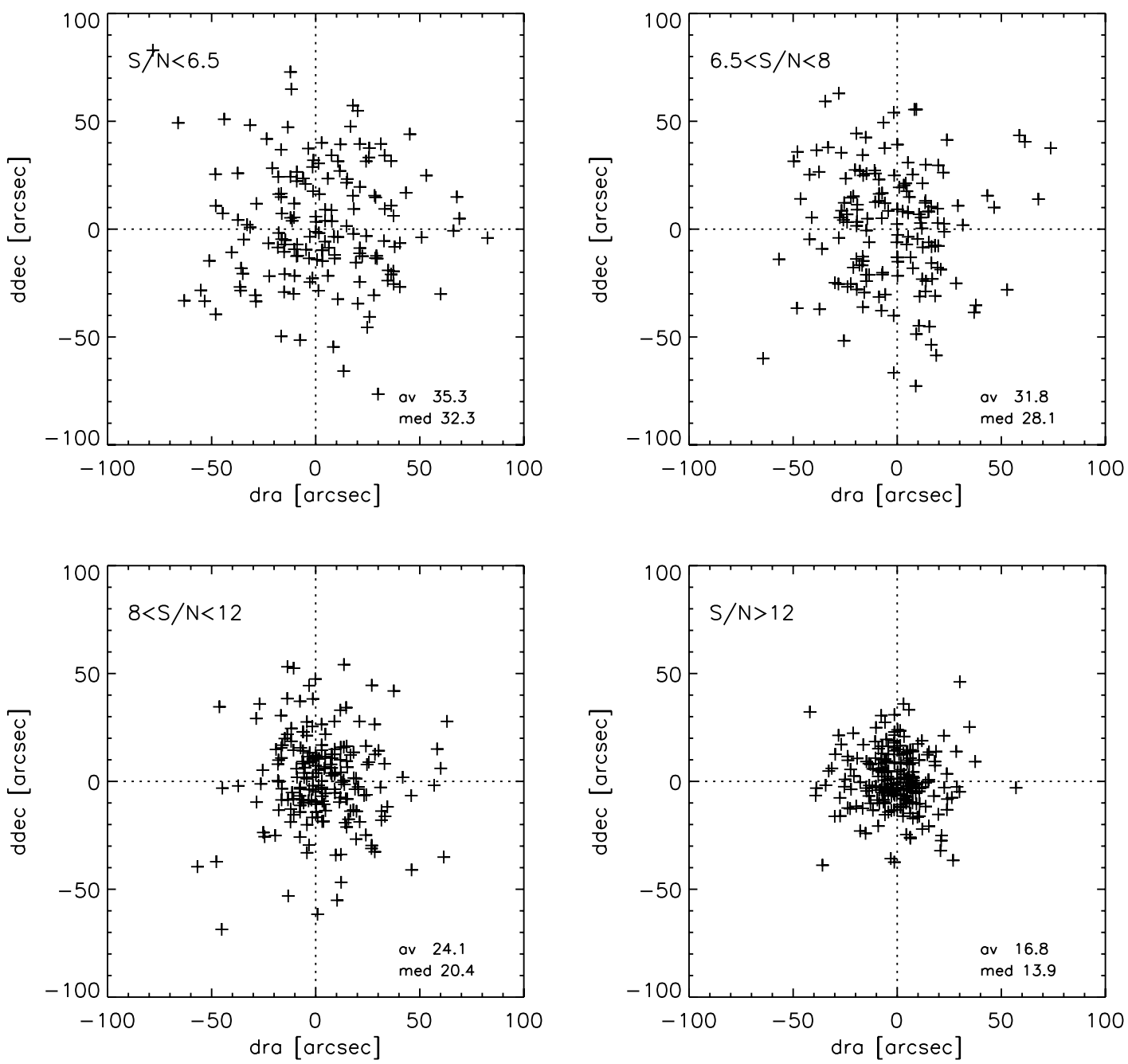

FIG. 8.-Differences between the positions of the $\mathrm{H}$ I sources, as indicated by the values in cols. (3) and (4) of Table 1, which are corrected for systematic telescope pointing errors, and the optical counterpart positions as listed in cols. (5) and (6) of Table 1. Sources are separated by S/ $\mathrm{N}$ as indicated in each panel. Average and median offsets, expressed in arcseconds, are listed within each panel.

by the present paper is strongly affected by local large-scale structure (the Virgo Cluster), as is evident in the redshift distribution presented in Figures 5 and 4. Detailed studies regarding environmental influences on $\mathrm{H}$ i content, the HIMF, and other characteristics will be incrementally enabled as further installments of ALFALFA data become available.

The principal conclusions which can be gleaned from this first installment of ALFALFA sources in a region covering $132 \mathrm{deg}^{2}$, which represents only $1.9 \%$ of the final survey, are as follows:

The ALFALFA survey is delivering high-quality $1.4 \mathrm{GHz}$ spectral line data as anticipated. The adopted "minimum intrusion" observing technique (Paper I) is highly successful at reducing spectral baseline instability, beam and sidelobe variations, and gain instabilities. Through a combination of deliberate calibration technique and empirical checks, the positional and photometric accuracy of extracted sources is meeting or even exceeding anticipated survey design specifications.

Although this region of the sky has been previously targeted heavily by surveys of optically selected galaxies (Gavazzi et al. 2003) and was covered by both the HIPASS (Wong et al. 2006) and HIJASS (Davies et al. 2004) Virgo Cluster surveys, 69\% of the $\mathrm{H}$ I detections presented here are new. The improvement over HIPASS in this region is a factor of 18 in the number density of $\mathrm{H}_{\mathrm{I}}$ detections. The galaxies detected by ALFALFA include a population of gas-rich, star-forming LSB galaxies that are not included in previous optical magnitude-limited surveys.

The median redshift of ALFALFA in this region of the sky is $\sim 7000 \mathrm{~km} \mathrm{~s}^{-1}$, This depth may be compared to that of HIPASS, $\sim 2800 \mathrm{~km} \mathrm{~s}^{-1}$ (Meyer et al. 2004). ALFALFA samples volumes well beyond the Local Supercluster, and the distribution of $\mathrm{H}$ I detections follows the large-scale structure evident in the region. Eventually, we will use the ALFALFA data set to measure cosmological parameters such as the clustering properties of the $\mathrm{H}_{\mathrm{I}}$ population and its bias parameter, and to explore further the "void problem" (Peebles 2001).

Twenty-seven of the $\mathrm{H}$ I sources are identified with galaxies of early morphology, types $\mathrm{E}, \mathrm{dE} / \mathrm{Sph}$, or S0. Half are in the vicinity of the Virgo Cluster and could be members. A study of the characteristics of these objects and the morphology and kinematics of the $\mathrm{H} \mathrm{I}$ is under way by R. Koopmann et al. (2007, in preparation).

Among the objects tabulated as $\mathrm{H}$ I detections here, several appear to be extended complexes of $\mathrm{H}$ I clouds. Referring to them by their AGC number (catalog number), 226054 (1-235), 
226055 (1-238), 226056 (1-239), and 224316 (1-241) are different clumps of the dark galaxy Virgo HI21 reported by Davies et al. (2004). The ALFALFA observations show that this feature is clearly connected to the nearby one-armed spiral NGC 4254; we discuss these observations in more detail elsewhere (M. P. Haynes et al. 2007, in preparation).

For $41 \mathrm{H}$ i sources, we have been unable to identify unambiguously their optical counterparts. More than half of these are found at redshifts less than $+200 \mathrm{~km} \mathrm{~s}^{-1}$ and are likely to be perigalactic HVCs. Particularly notable among them are the compact HVCs with positive velocities, a peculiar population in the vicinity of the north Galactic pole. A number of the objects reported by de Heij et al. (2002) with fluxes close to the detection limit of that survey are not confirmed by the more sensitive ALFALFA observations (B. R. Kent et al. 2007, in preparation). The objects with positive velocity that are confirmed, and a few previously undetected with similar velocities, lie in the same sky region as several distant perigalactic structures exhibiting positive velocities: the Bootes dwarf spheroidal galaxy (Belokurov et al. 2006a; Muñoz et al. 2006), the leading arm of the Sagittarius stream and others found in the "Field of Streams" (Belokurov et al. 2006b), and farther away but still at $\sim 200 \mathrm{~km} \mathrm{~s}^{-1}$, the Local Group galaxy GR 8 . We are in the process of investigating the interpretation of these features in more detail.

For some of the remaining objects without clear optical identifications, possible optical counterparts exist, but the positional accuracy of the ALFALFA $\mathrm{H}$ I data is insufficient to yield an unambiguous identification. Interestingly, 17 of the candidate $\mathrm{H}_{\mathrm{I}}$ detections with no discernible optical counterpart are unlikely to be HVCs. In the cases of AGC 215217 (1-62), AGC 226061 (1257), AGC 226080 (1-316), and AGC 223449 (1-271), galaxies are seen within few arcminutes of the $\mathrm{H}$ i positions, which have redshifts comparable with those of the $\mathrm{H}$ I features. AGC 226080 has been shown by Oosterloo \& van Gorkom (2005) to be a plume extending from NGC 4388. AGC 223449 may have very low $\mathrm{H}$ I mass, although its distance is highly uncertain. It remains to be verified whether a connection between the optical and $\mathrm{H}_{\mathrm{I}}$ objects exists. In the case of AGC 225998 (1-173), another object of highly uncertain distance, a H I-rich galaxy (UGC 7235, NGC 4189, 1-175) is found at comparable redshift, but half a degree away. AGC 226118 (1-354) could be an interesting feature in the Virgo Cluster, but its proximity to M87 makes the detection doubtful in spite of a better than fair S/N. AGC 215230 (1-44), AGC 226043 (1-185), AGC 226117 (1-352), AGC 226119 (1-360), and AGC 226120 (1-366) have velocities in excess of $4000 \mathrm{~km} \mathrm{~s}^{-1}$; most in that group are marginal candidate detections. All others sources have identified optical counterparts, thanks to the good positional accuracy of ALFALFA source candidates. A preliminary analysis of Virgo Cluster H I sources with no optical counterparts is given in B. R. Kent et al. (2007, in preparation).

In summary, we present the first catalog release of the ALFALFA survey, corresponding to $1.9 \%$ of the projected sky coverage of the completed survey. As anticipated, ALFALFA delivers a dramatic improvement in $\mathrm{H}$ I detection sensitivity over previous $\mathrm{H}_{\mathrm{I}}$ blind surveys through its combination of wide areal coverage, smaller beam area, higher spectral resolution, and the sheer sensitivity superiority offered by Arecibo's huge collecting area. The vast majority of $\mathrm{H}$ I sources listed in the present catalog have identified optical counterparts. Many are vigorously star-forming yet optically faint, late-type galaxies. Among those H I sources that cannot be unambiguously identified with an optical galaxy, we find a population of HVCs, including ones at significant positive velocity, and a few legitimately extragalactic objects whose optical counterparts are not yet identified.

In addition, the survey identifies nearly as many candidate detections of lower $\mathrm{S} / \mathrm{N}$, including many with narrow spectral lines and no optical counterparts. Because candidate source reliability plummets below $\mathrm{S} / \mathrm{N}<6.5$, these $\mathrm{H}$ I candidates are not reported here but are targeted for corroborating follow-up observations with the Arecibo L-band wide system employing an efficient strategy designed for that purpose, as discussed in Paper I.

A catalog of comparable size, covering a strip of the same R.A. extent and including the southern part of the Virgo Cluster, is in preparation (B. R. Kent et al. 2007, in preparation); eventually, ALFALFA will cover the entire region included in the Virgo Cluster Catalog (Binggeli et al. 1985). Catalog data releases for other parts of the sky are also in preparation by several groups within our collaboration, as are programs involving multiwavelength follow-up studies of selected targets presented here. The catalog presented here and its associated data products will be incorporated into the more extensive digital $\mathrm{H}$ I data set as part of the ALFALFA Arecibo legacy (see footnote 15).

R. G., M. P. H., N. B., T. B., and R. K. acknowledge the partial support of NAIC as Visiting Scientists during the period of this work. This work has been supported by NSF grants AST 03-07661, AST 04-35697, AST 03-47929, AST 04-07011, and AST 03-02049 and by a Brinson Foundation grant. We thank the Director of NAIC, Robert Brown, for stimulating the development of major ALFA surveys, Héctor Hernández for his attention to the telescope scheduling, and the Director, telescope operators, and support staff of the Arecibo Observatory for their proactive approach. We also thank Tom Shannon for his advice and assistance with hardware, system, and network issues at Cornell.

\section{REFERENCES}

Barnes, D. G., et al. 2001, MNRAS, 322, 486

Belokurov, V., Evans, N. W., Irwin, M. J., Hewett, P. C., \& Wilkinson, M. I. 2006a, ApJ, 637, L29

Belokurov, V., et al. 2006b, ApJ, 642, L137

Binggeli, B., Sandage, A., \& Tammann, G. A. 1985, AJ, 90, 1681

Chamaraux, P., Balkowski, C., \& Gerard, E. 1980, A\&A, 83, 38

Dale, D. A., \& Giovanelli, R. 2000, in ASP Conf. Ser. 201, Cosmic Flows

Workshop, ed. S. Courteau \& J. Willick (San Francisco: ASP), 25

Davies, J., et al. 2004, MNRAS, 349, 922

Davies, R. D., \& Lewis, B. M. 1973, MNRAS, 165, 231

de Heij, V., Braun, R., \& Burton, W. B. 2002, A\&A, 391, 159

Gavazzi, G., Boselli, A., Donati, A., Franzetti, P., \& Scodeggio, M. 2003, A\&A, 400,451
Giovanelli, R., \& Haynes, M. P. 1983, AJ, 88, 881

Giovanelli, R., Haynes, M. P., Freudling, W., da Costa, L. N., Salzer, J. J., \& Wegner, G. 1998, ApJ, 505, L91

Giovanelli, R., et al. 2005a, AJ, 130, 2598 (Paper I) . 2005b, AJ, 130, 2613 (Paper II)

Masters, K. L., Haynes, M. P., \& Giovanelli, R. 2004, ApJ, 607, L115

Meyer, M. J., et al. 2004, MNRAS, 350, 1195

Minchin, R., et al. 2005, ApJ, 622, L21

Muñoz, R. R., Carlin, J. L., Frinchaboy, P. M., Nidever, D. L., Majewski, S. R., \& Patterson, R. J. 2006, ApJ, 650, L51

Oosterloo, T., \& van Gorkom, J. 2005, A\&A, 437, L19

Peebles, P. J. E. 2001, ApJ, 557, 495

Rosenberg, J. L., \& Schneider, S. E. 2002, ApJ, 567, 247 
Saintonge, A. 2007, AJ, 133, 2087

Shostak, G. S., \& Allen, R. J. 1980, A\&A, 81, 167

Skrutskie, M. F., et al. 2006, AJ, 131, 1163

Solanes, J.-M., Sanchis, T., Salvador-Solé, E., Giovanelli, R., \& Haynes, M. P. 2002, AJ, 124, 2440

Springob, C. M., Haynes, M. P., Giovanelli, R., \& Kent, B. R. 2005, ApJS, 160, 149
Springob, C. M., Masters, K. L., Haynes, M. P., Giovanelli, R., \& Marinoni, C. 2006, ApJS, submitted

Wong, O. I., et al. 2006, MNRAS, 371, 1855

York, D. G., et al. 2000, AJ, 120, 1579 